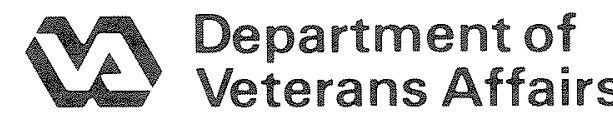

Journal of Rehabilitation Research

and Development Vol. 27 No. 3, 1990

Pages 255-278

\section{A test suite for hearing aid evaluation}

\author{
James M. Kates, EE \\ Center for Research in the Speech and Hearing Sciences, City University of New York, Graduate School and \\ University Center, New York, NY 10036
}

\begin{abstract}
A test suite has been developed for evaluating hearing aids. The tests in the suite are frequency response, number of processing bands and type of processing, input/output characteristics, processing attack and release times, and broadband distortion. The test suite produces a more complete evaluation of a hearing aid than any previous set of tests, and is suitable for the automatic evaluation of a hearing aid containing unknown processing. The test procedures are described, and sample test results are presented for simulated linear and two-channel compression hearing aids.
\end{abstract}

Key words: amplifier, automatic gain control, automatic signal processing, frequency response, hearing aid, sound pressure level, test suite.

\section{INTRODUCTION}

A test suite has been developed for the evaluation of hearing aids and related devices. The test suite is designed to measure the characteristics of existing hearing aids and to accommodate new processing technology as it develops. The test procedures identify the signal-processing parameters of an instrument and measure overall performance.

An effective test procedure must work with the many types of signal processing available in hearing aids. The most common instruments are single-channel, containing linear processing that shapes the frequency response and provides gain. Automatic gain control (AGC) is available

Address all correspondence and reprint requests to: James M. Kates, EE, Center for Research in the Speech and Hearing Sciences, CUNY, Graduate School and University Center, Room 901, 33 West 42nd Street, New York, NY 10036. in some hearing aids, typically as a circuit that monitors the input signal level and provides linear gain for an input below the selected threshold and a fixed limiting output level for a signal above the threshold. Newer signalprocessing circuits, often termed automatic signal processing (ASP), can adjust the frequency response of a hearing aid in response to the spectrum and level of the incoming signal (14). More complicated instruments, having two or more signal-processing channels that may contain independent compression circuits with adjustable compression thresholds and compression ratios, are being introduced and are expected to become prevalent over the next several years.

Test procedures, however, often lag behind the advances in hearing aid technology. The manufacturers' specifications for a hearing aid, for example, are commonly determined in accordance with the hearing aid test procedures given in American National Standards Institute ANSI S3.22 (1). The ANSI standards are not meant to be allinclusive; rather, they are primarily quality control standards for the manufacture of the instruments and were originally developed for single-channel hearing aids containing linear or compression processing. More complicated processing, such as ASP or two-channel compression, cannot be adequately tested using the ANSI S3.22 procedures. Thus new tests are needed for the evaluation of the newest generation of hearing aids.

The characterization of a hearing aid requires a series of tests, since no one test can give a complete description of how the processing behaves. In developing the test suite, existing tests have been used where relevant and new tests have been derived where existing tests are inadequate. A 
Journal of Rehabilitation Research and Development Vol. 27 No. 3 Summer 1990

total of five tests make up the test suite:

1. Frequency response as a function of input level

2. Type of processing and number of bands

3. Input/output characteristics for each band

4. Attack and release times for each band

5. Broadband distortion as a function of input level.

Shaped broadband noise signals are used for the frequency-response and distortion tests, and noise combined with a swept bias tone is used for determining the type of processing. Sinusoids stepped in amplitude are used for the input/output and attack/release measurements, with the test-frequency selection based on the results of the determination of the number and spacing of the processing bands.

The test suite has been implemented on a generalpurpose personal computer as a set of programs that generate the test signals and analyze the hearing aid response. A complete hearing aid test system would require the addition of a digital-to-analog (D/A) converter and amplifier to apply the test signal to the hearing aid, an acoustic test box, and an amplifier and analog-to-digital (A/D) converter to acquire the hearing aid response in a form suitable for digital analysis. Such a test system is being built as part of a related project. In the meantime, the test suite has been evaluated using a digital simulation of a two-channel hearing aid having independent compression in each channel and including amplitude clipping in the amplifier.

The purpose of this paper is to describe the test suite and to illustrate the analysis results for typical hearing aids. The paper starts with a description of each of the five tests, including the objective of the test, the test signal and method of generation, and the signal-processing procedure for the analysis. The parameters of the simulated linear and twochannel compression hearing aids used to illustrate the tests are then presented. This is followed by a presentation of the test results, showing the test performance and indicating how hearing aid processing and adjustments can affect the analysis.

\section{TEST PROCEDURES}

The hearing aid test procedures are designed to form a practical computer-based measurement system. The concern with a practical implementation is reflected in the assumed accuracy of the D/A and A/D converters. Since the greatest level of accuracy readily available in computerbased data-acquisition systems is 16 bits, all of the procedures have been designed to work with 16-bit $\mathrm{A} / \mathrm{D}$ and $\mathrm{D} / \mathrm{A}$ converters. The simulation results presented here include 16-bit quantization of the test signals and hearing aid output.

The test signals span a range from 60 through $90 \mathrm{~dB}$ sound pressure level (SPL), and these signals have to fit within the dynamic range limitations of the 16 -bit $\mathrm{D} / \mathrm{A}$ converter. The assumed maximum output of the system without saturating the D/A converter is a sinusoid having a level of $102 \mathrm{~dB}$ SPL, which gives enough dynamic range for the output of a $90 \mathrm{~dB}$ SPL noise signal without clipping, given a crest factor (peak-to-average ratio) of approximately $12 \mathrm{~dB}$. The dynamic range allowance for the most intense signals means that lower-level test signals will show some quantization distortion, although this will typically be below the noise level of the hearing aid under test.

Since the maximum power output (MPO) of different hearing aids can cover quite a wide dynamic range, the measurement system will need an attenuator to match the output of the hearing aid under test to the available dynamic range of the 16-bit A/D converter in the measurement system. The amount of attenuation needed to match the maximum hearing aid output signal to the full-scale input level of the A/D converter can be determined at the beginning of the set of tests and stored to give a scale factor for adjusting the response curves.

A related concern is the sampling rate used for the signal generation and analysis. The bandwidth of most hearing aids is limited by the receiver, which typically rolls off rapidly above 5 or $6 \mathrm{kHz}$ (12). Thus, a sampling rate of $20 \mathrm{kHz}$ was chosen to provide an adequate frequency range for a typical instrument; a higher sampling rate would require more processing time, but would not yield much additional information. Hearing aids with a bandwidth exceeding approximately $8 \mathrm{kHz}$, however, would require a higher sampling rate.

In order for the system to be practical, the processing time must also be kept within reasonable limits. In terms of the algorithms, this has resulted in the selection of singlechannel analysis, based on the power spectrum of the hearing aid output, as opposed to two-channel analysis based on the cross-correlation of the output signal with the input signal. As compared to the two-channel analysis, the single-channel analysis requires half the amount of computations for a given number of data samples. A further consequence is that short test sequences have been used in order to minimize the processing time. The objective is that the complete test suite can be run in less than 20 minutes.

\section{Frequency response}

The frequency-response measurements use the shaped 
Gaussian noise signal described by Burnett, et al. (5). The stimulus consists of white Gaussian noise that has been band-limited to the range $200 \mathrm{~Hz}$ to $5000 \mathrm{~Hz}$, and then shaped with a one-pole low-pass filter at $900 \mathrm{~Hz}$ to give an approximate match to the long-term spectrum of speech. This signal is intended to test the hearing aid under conditions similar to excitation by speech. While it is obvious that the envelope fluctuations of speech are not reproduced, this test signal does come closer to a speech-like stimulus than the single swept sinusoid specified in the ANSI S3.22 standard (1).

A second reason for the use of shaped noise is to avoid the phenomenon of "blooming" that is often observed when an AGC instrument is tested with a swept sinusoid. The problem, as pointed out by Preves, et al. (19), is that a swept sinusoid at a constant amplitude may activate the AGC circuitry over only part of the frequency range. As the input signal level is increased, the behavior of the instrument may appear to be linear at low frequencies, where the signal level is below the AGC threshold, and compressive at high frequencies, where the AGC threshold is lower due to the frequency selectivity built into a typical AGC control circuit. Thus the frequency response appears to emphasize low frequencies as the input level is increased.
The use of a broadband stimulus avoids this measurement artifact, since all frequencies are present simultaneously.

The test signal is generated from a digital pseudorandom noise (PN) generator. This gives a set of uniform random deviates between 0 and 1 , which are then transformed into samples from a normal (Gaussian) distribution having zero mean and a specified output power. The white Gaussian noise is then convolved with the impulse response of the shaping filter. The filter is a digital version of the analog filter used by Burnett, et al. (5), and is derived in Appendix A. The frequency response of the digital filter is shown in Figure 1. The test signal is used at 60 through $90 \mathrm{~dB}$ SL input intensities in steps of $10 \mathrm{~dB}$.

The hearing aid output is analyzed using the method of modified periodigrams (21). A total of 40,960 samples $(2.05 \mathrm{~s})$ are taken of the hearing aid response to the shaped noise. The first 4,096 samples ( $205 \mathrm{~ms}$ ) are discarded, since these may contain transients, and the remaining samples are processed in blocks of 1,024 samples ( $51.2 \mathrm{~ms}$ ), using a Hanning window and 50 percent overlap. The Hanning window gives more accurate results in regions of low relative spectral energy than other commonly used window shapes (e.g., Hamming) when used for this application. The resultant estimated power spectrum is then smoothed

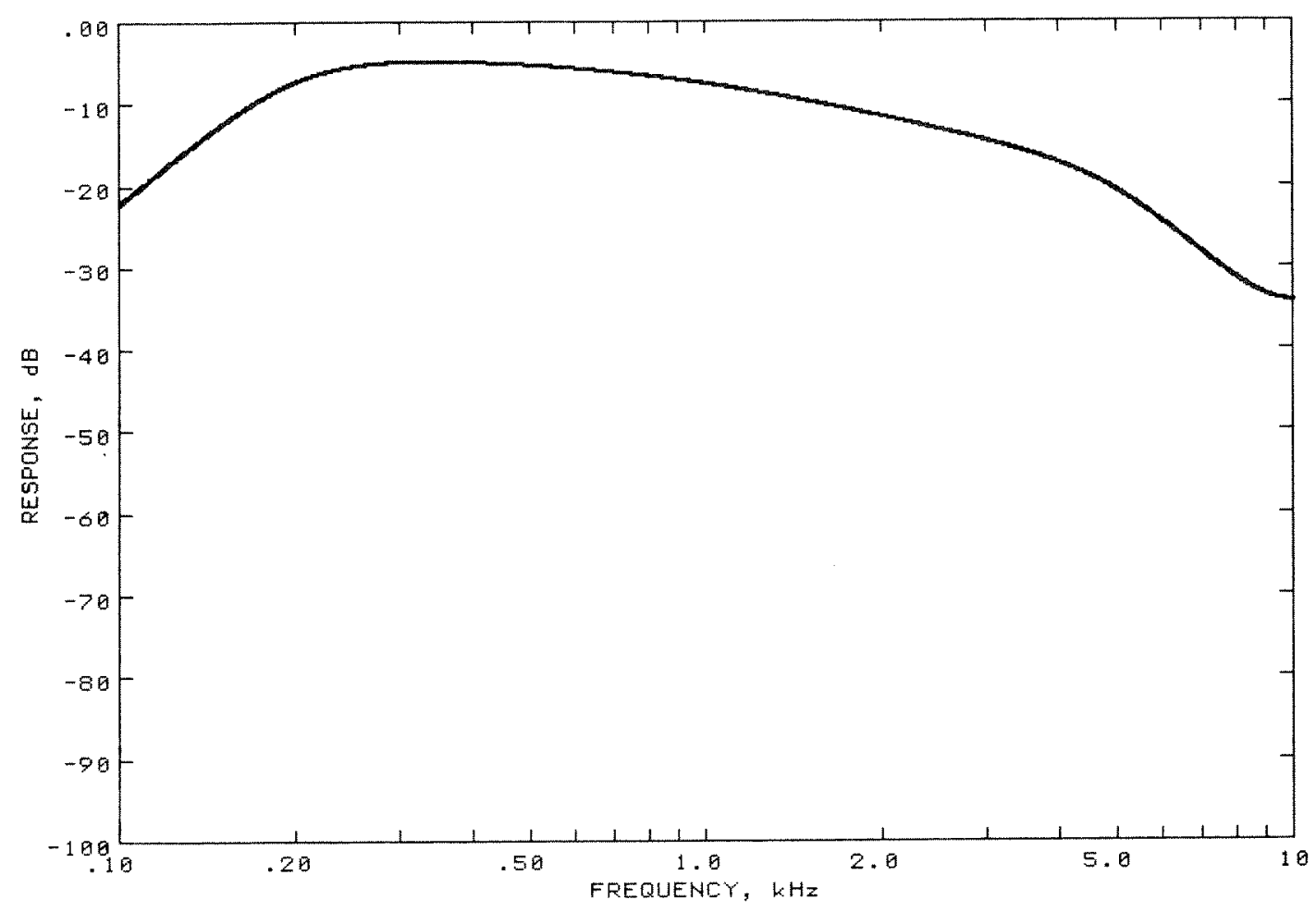

Figure 1.

Power spectrum of the digital shaped noise test signal. 
Journal of Rehabilitation Research and Development Vol. 27 No. 3 Summer 1990

by combining the frequency points into overlapping onethird octave bands, which reduces the variance in the measurement, although it will broaden sharp spectral peaks in the hearing aid frequency response. The power spectrum is divided by the spectrum of the noise-shaping filter, also smoothed by one-third octave bands, to give the gain of the hearing aid as a function of frequency. The level of the input signal in $\mathrm{dB}$ is then added to the gain in $\mathrm{dB}$ of the hearing aid to get a family of offset gain curves.

\section{Processing type}

The type of processing in the hearing aid is determined by observing how a bias tone modifies the frequency response of the instrument. To this end, the response of the instrument is measured for an excitation consisting of the $60 \mathrm{~dB}$ SPL-shaped noise combined with an $80 \mathrm{~dB}$ SPL swept sinusoid. The response of the instrument to the noise alone and to the swept tone alone are also measured and stored. The purpose of the swept tone is to bias the nonlinear processing that may be present in the hearing aid; the energy in the swept tone is detected by the control circuitry which then changes the hearing aid response. The shaped noise signal is used to determine the frequency response of the hearing aid while these changes take place.

As the swept bias tone moves through different frequency regions, it will change the gain and/or frequency response of a hearing aid containing nonlinear processing such as AGC, ASP, or amplifier saturation. Most commercially available single-channel AGC hearing aids, for example, have a bandpass filter tuned to the region around $2 \mathrm{kHz}$ in front of the compressor control circuit and a threshold above $65 \mathrm{~dB}$ SPL in the most sensitive frequency region. An input at $80 \mathrm{~dB}$ SPL will be processed linearly if it is far enough removed from $2 \mathrm{kHz}$, but will cause a reduction in gain if it is near $2 \mathrm{kHz}$. The swept tone thus reduces the gain while it is present in the frequency region or regions that control the nonlinear processing. The gain will be unchanged from the linear value, however, when the swept tone is either too low or too high in frequency to be detected by the control circuitry.

To determine the changes in the frequency response caused by the nonlinear processing, the hearing aid response to the swept tone alone is subtracted from the response to the swept tone plus noise. This leaves the shaped-noise output as modified by the presence of the sweep. The spectrum of this noise is then compared to the spectrum of the hearing aid response to the shaped noise alone; the difference in the spectra indicates the degree of nonlinear processing in the hearing aid caused by the presence of the bias tone. The changes in the spectral difference as a function of the sweep frequency indicate the frequency regions controlling the nonlinear processing. A linear hearing aid will result in no recorded gain changes at any frequency of the swept tone; different realizations of equivalent linear processing, such as singlechannel or multi-channel frequency shaping, will thus be indistinguishable by this procedure.

The changes in gain and frequency response are determined in 12 third-octave bands spaced from 315 to 4000 $\mathrm{Hz}$ at the ANSI center frequencies (2). The swept tone spends a time equivalent to 4,096 samples ( $205 \mathrm{~ms}$ ) moving across each third-octave band, so the instantaneous sweep frequency has an exponential dependence on time, since the width of the bands increases with increasing center frequency. The sweep starts one third-octave below $315 \mathrm{~Hz}$, with the first 4,096 samples (205 ms) discarded because of the possibility of transients, and then moves through the 12 desired bands. A total of 53,248 samples ( $2.66 \mathrm{~s})$ are therefore analyzed, of which the last 49,152 (2.46 s) are used in determining the type of processing present in the hearing aid. The equations describing the sinusoidal sweep are given in Appendix B; the frequency extent of the swept tone can obviously be modified if needed.

A block diagram of the signal processing system is shown in Figure 2. As described above, three separate signals are sent through the hearing aid: the noise alone at $60 \mathrm{~dB}$ SPL, the swept tone alone at $80 \mathrm{~dB}$ SPL, and the shaped noise and swept tone combined. The hearing aid response to each of these signals is sampled and stored in the computer. An adaptive Widrow $(22,23)$ noise cancellation procedure, modified for multiple reference signals (9) and adaptive estimation of the signal energy (11), is then used to estimate the changes in the system frequency response caused by the swept bias tone. The adaptive processing is described in more detail in Appendix $\mathbf{C}$.

The adaptive noise cancellation algorithm simultaneously adjusts the filters $w(n)$ and $v(n)$ shown in Figure 2 to minimize the power in the output error signal. The input to filter $w(n)$ is the sinusoidal sweep, so very little adjustment should be needed to get nearly perfect cancellation of the sinusoidal portion of the combined signal. This adaptive filter is provided, however, since there may be situations where the presence of the shaped noise modifies the response of the hearing aid to the swept tone. The input to filter $\mathrm{v}(\mathrm{n})$ is the hearing aid response to the noise alone, so the filter $v(n)$ has to adjust the noise-alone response to cancel the noise response in the presence of the bias sweep. The process of filtering the noise-alone response to match the noise response in the presence of the swept tone means 


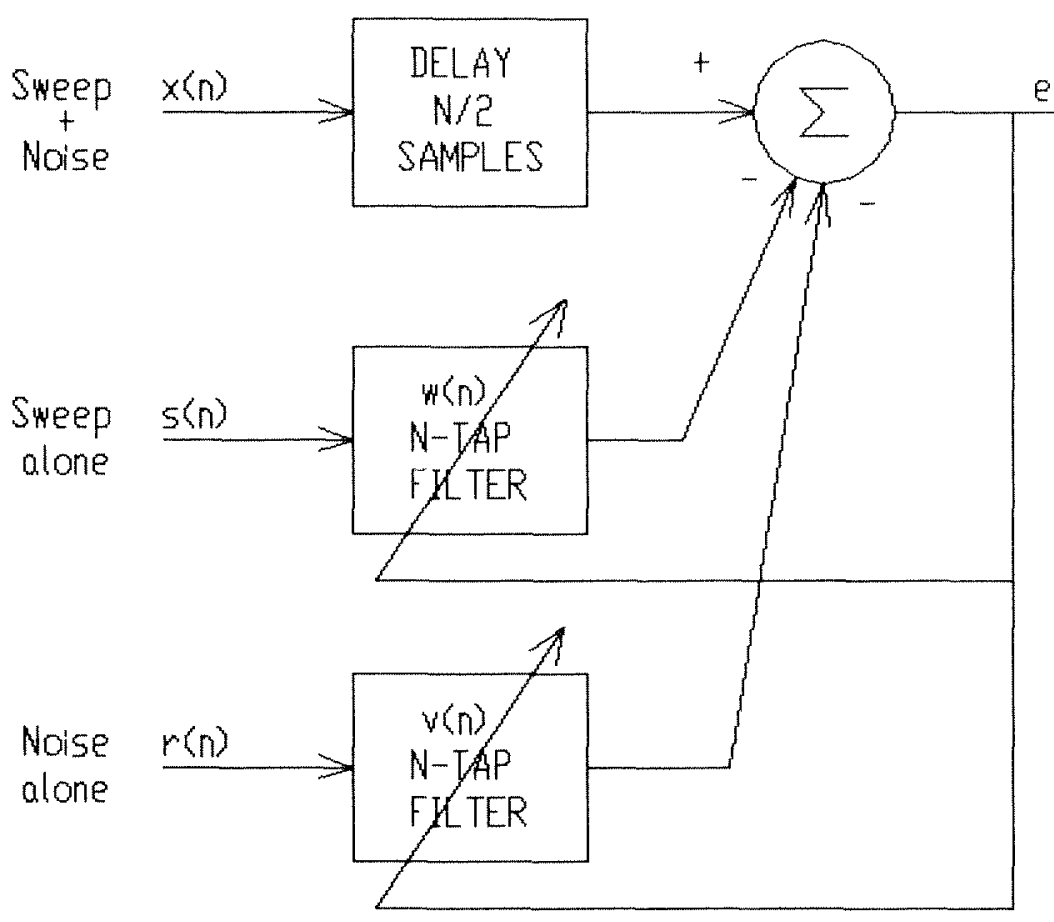

Figure 2.

Adaptive system for determining the type of processing.

that filter $v(n)$ directly provides an estimate of the gain and frequency-response changes caused by the swept sinusoid.

The length of the adaptive filters represents an engineering compromise. A short filter will adapt more rapidly to rapid gain changes in the hearing aid, but a long filter will give better frequency resolution. For the test sequence length given above being used to test a twochannel instrument, a filter length of 15 taps has been found to be adequate. For more channels in the hearing aid, both the adaptive filter length and the duration of the test sequence should be increased so that more time is spent traversing each third-octave band.

The coefficients of filter $v(n)$ are sampled each time the swept tone crosses the boundary of one of the 12 thirdoctave frequency bands and again at the end of the sweep. The frequency response is computed for each set of filter coefficients, giving a total of 13 plots. In order to simplify the presentation, the frequency response is calculated only at the frequencies corresponding to each edge of the thirdoctave bands. It is replaced by a blank if the reduction in gain is less than $3 \mathrm{~dB}$, and by a solid character if the reduction in gain is equal to or greater than $3 \mathrm{~dB}$. This leads to a square two-dimensional grid in which the gain change in each third-octave frequency region is indicated each time the sweep crosses a third-octave boundary.

A set of idealized system-identification patterns is presented in Figure 3 for a one- or two-channel hearing aid with various types of signal processing. A perfectly linear system, as shown in the upper left-hand pattern, results in a blank pattern because the swept tone does not cause any gain change at any frequency.

The next pattern is for an ASP circuit (14). In this type of processing, the cutoff frequency of a high-pass filter moves lower in frequency as the amount of low-frequency energy decreases. The detection circuit that controls the high-pass filter tends to be most sensitive to energy around $250-300 \mathrm{~Hz}$. This processing leads to a pattern in which the lower left-hand corner is blacked out on a diagonal, because raising the sweep frequency causes a reduction in the amount of energy detected by the control circuit, and as a result, the ASP cutoff frequency moves lower.

A broadband AGC circuit of the type discussed in this section results in the pattern shown in the upper right-hand corner of Figure 3. This pattern has a horizontal stripe blacked out since the gain at all frequencies is reduced as soon as the sweep goes above the threshold most sensitive to energy at $2 \mathrm{kHz}$. The processing is linear at low frequencies since the energy detected by the control circuit is still below the AGC threshold. Combining ASP and AGC results in a pattern where both the ASP and the AGC areas are blacked out, is shown in the lower left-hand corner.

A two-channel hearing aid having compression only in the lower frequency channel results in the next pattern. When the swept tone is in the low-frequency channel, the AGC detects the energy and causes a reduction in the gain in the channel. The gain in the high frequency is un- 
Journal of Pehabilitation Research and Development Vol. 27 No. 3 Summer 1990
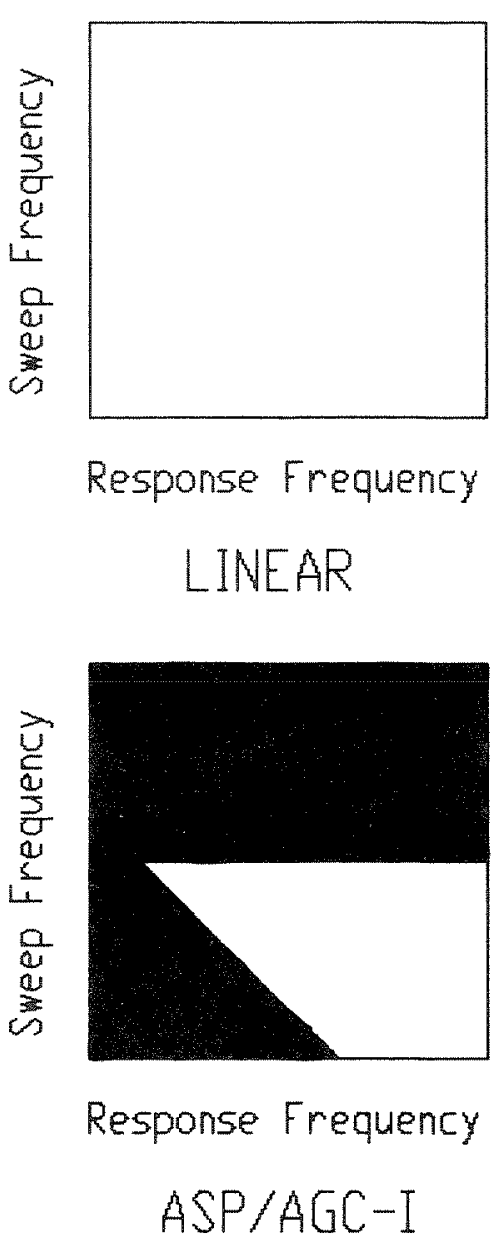
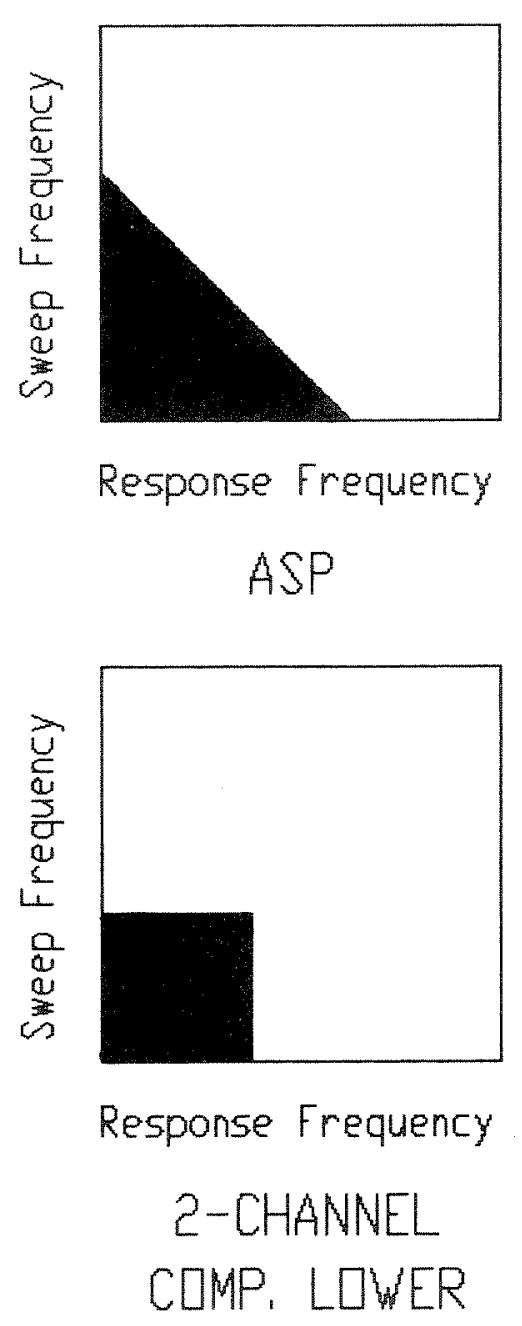
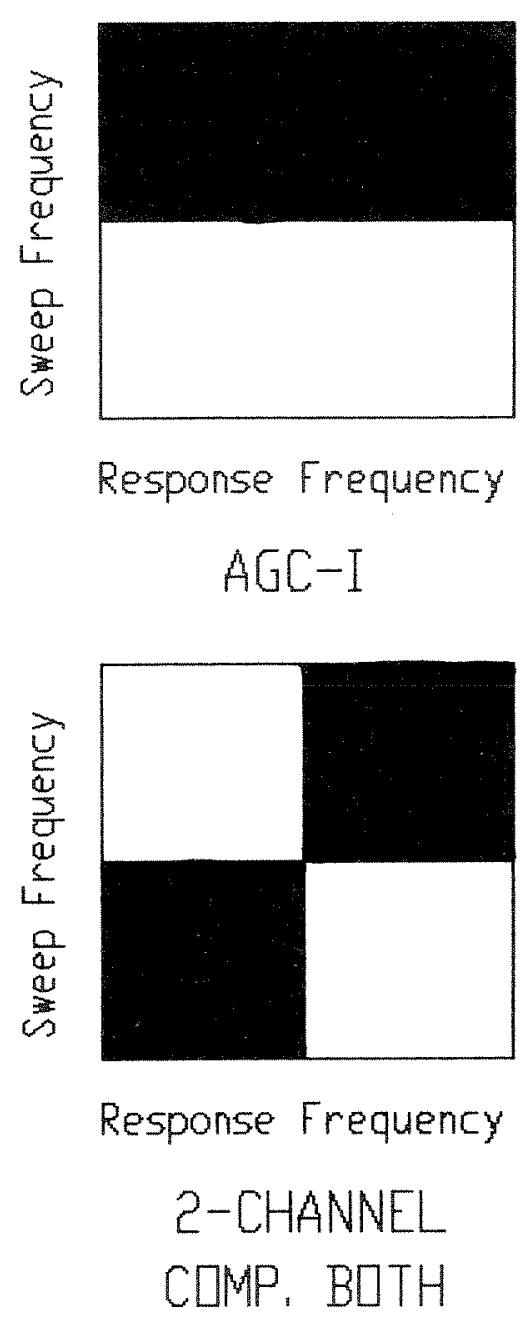

Figure 3.

Idealized system identification patterns for determining the type of processing.

changed. Thus the lower left-hand corner is blacked out because the gain at low frequencies is reduced as long as the sweep is in the lower frequency channel. The lowfrequency gain returns to the linear value as soon as the sweep moves out of the low-frequency channel.

A two-channel instrument having compression in both channels results in the checker-board pattern shown in the lower right-hand corner of Figure 3. The gain at low frequencies is reduced while the sweep is in the low-frequency channel. The gain at high frequencies is kept at the linear value since the $\mathrm{AGC}$ in that channel has not been engaged. The gain at high frequencies is then reduced when the sweep moves into the high-frequency channel, and the gain at low frequencies returns to the linear value for the channel.

The test program correlates the actual system identification pattern for the hearing aid under test with each of the idealized patterns shown in Figure 3 to return a patternmatch score. The correlation gives a value of 1 at an individual point if the actual and idealized patterns both have the same gain characteristic. It gives a value of -1 if one pattern shows no gain reduction but the other pattern does show a gain reduction at the point. The values are summed over the entire pattern and normalized by the number of points to give the test scores, which range from 1 to -1 . In determining the scores for the processing options, all possible crossover frequencies are tried for each option, with the program indicating the score for the best frequency match and the crossover frequency at which it occurs. The final crossover frequency estimated by the test program is then the crossover frequency at which the highest test score was found, and the indicated processing is that which corresponds to the highest correlation score. 


\section{Input/output characteristics}

In a multichannel hearing aid, the input/output characteristics are measured at or near the geometric center of each frequency band. In a single-channel instrument the measurement is made at the $2 \mathrm{kHz}$ frequency used for the ANSI attack and release time measurements (1) because many single-channel compression instruments are most sensitive at this frequency. The geometric center of the lowfrequency band is determined as the square root of the product of the crossover frequency and $315 \mathrm{~Hz}$, and the geometric center of the high-frequency band is determined as the square root of the product of the crossover frequency and $4 \mathrm{kHz}$. The actual test frequencies are then the ANSI third-octave frequencies (2) closest to the computed band centers.

The test signal is a sinusoid of fixed frequency having segments stepped in level from $40 \mathrm{~dB}$ SPL to $95 \mathrm{~dB}$ SPL in steps of $5 \mathrm{~dB}$. The duration of each segment is $250 \mathrm{~ms}$ (5,000 samples), with the first $100 \mathrm{~ms}$ an allowance for any transients to stabilize and the signal amplitude determined from the average over the last $150 \mathrm{~ms}$ of the segment. The test signal has 12 segments, so the total signal duration is $3 \mathrm{~s}$ (60,000 samples). The input level, the output level, and the compression ratio are indicated for each of the segments.

In order to reduce the amount of computer processing, the test signals are generated in advance and stored on disc. This precludes generating a test signal at each arbitrary band center frequency; instead, the precomputed signal closest to the desired center frequency is used. A set of 12 test signals, spaced at the ANSI standard (2) third-octave frequencies between $315 \mathrm{~Hz}$ and $4000 \mathrm{~Hz}$ inclusive, appears to be adequate. Note that the ANSI standard (1) test frequency of $2000 \mathrm{~Hz}$ is part of this set.

\section{Attack and release times}

The test procedure for measuring the attack and release times is based on the ANSI standard (1), but with provision made for analysis at the third-octave frequency closest to the geometric center of each hearing aid processing band. The test signal starts at a level of $55 \mathrm{~dB}$ SPL, jumps to $80 \mathrm{~dB}$ SPL after $200 \mathrm{~ms}$ (4,000 samples), and returns to $55 \mathrm{~dB}$ SPL at $600 \mathrm{~ms}(12,000$ samples $)$. The total signal duration is $1,000 \mathrm{~ms}$ (20,000 samples), but this can be lengthened for an instrument with unusually long attack or release times. The attack time is the time it takes for the hearing aid output to decay to a level $2 \mathrm{~dB}$ above the steady-state level after the test signal jumps to $80 \mathrm{~dB}$ SPL. The release time is the time it takes for the hearing aid output to reach a level $2 \mathrm{~dB}$ below the steady-state level after the test signal returns to the $55 \mathrm{~dB}$ SPL level.

Measuring the attack and release times requires extracting the envelope of the signal. Because the test signal is at a constant frequency, the simple system of Figure 4 is appropriate. The all-pass filter is a one-zero/one-pole filter, adjusted to give 90 degrees of phase shift at the test frequency. The direct signal path thus gives the in-phase part of the signal envelope, while the output of the all-pass filter provides the quadrature component. Squaring and summing the two signals results in the magnitude-squared signal envelope used in determining the attack and release times.

\section{Broadband distortion}

The ANSI standard (1) distortion measurement recommends total harmonic distortion for input sinusoids at 500 , 800 , and $1600 \mathrm{~Hz}$. This type of measurement does not give any indication of how distortion at high frequencies, where many hearing aids have their highest amount of gain, and therefore most easily go into amplifier saturation, will affect the reproduction of lower frequency speech sounds occurring at the same time. A procedure for measuring intermodulation distortion for a broadband stimulus, on the other hand, can provide information on the amount of

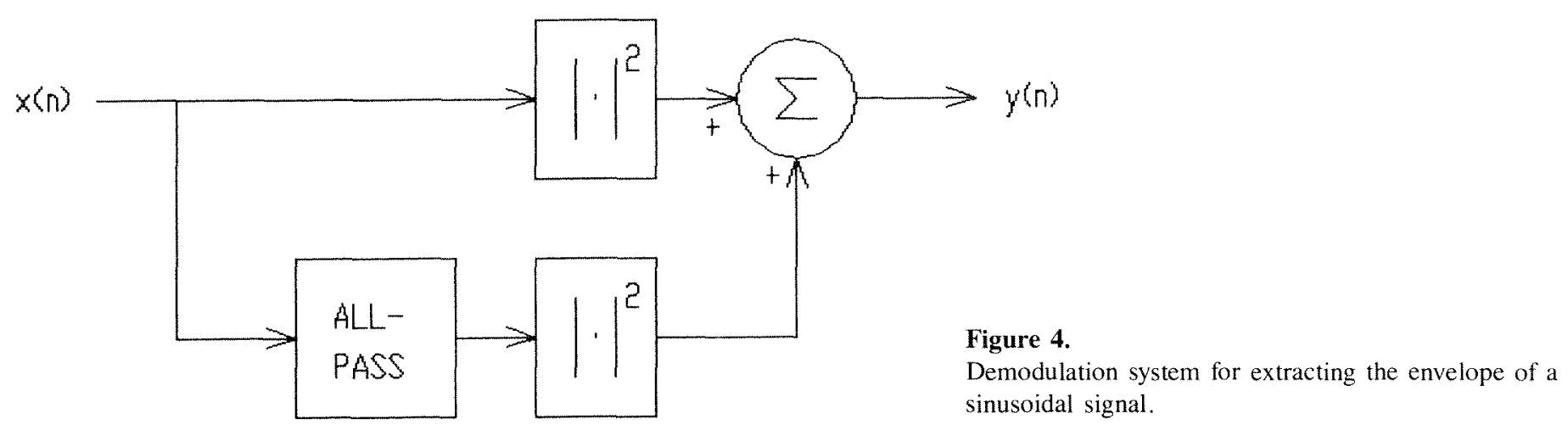


Joumal of Rehabilitation Research and Development Vol. 27 No. 3 Summer 1990

distortion that will occur under typical listening conditions.

Noise distortion measurements have been proposed before $(4,18)$. These procedures used a broadband stimulus convolved with an analog filter having a single notch in the transfer function. The noise distortion was computed from the amount of energy that was found in the notch. The system used in the test suite differs from these earlier proposals in that multiple notches are placed in the test signal rather than the single notch, and the test-signal generation is based on digital signal processing techniques.

The test signal is the shaped noise used for the frequency-response measurements and which is then convolved with a comb filter to create a series of interleaved peaks and valleys. The comb filter is a half-band filter designed using the Parks-McClellan design program (16) and having $0 \mathrm{~dB}$ pass-band gain and $62 \mathrm{~dB}$ stop-band attenuation, so it is theoretically possible to measure distortion values of less than 0.1 percent. Replacing the unit delay in the half-band high-pass filter with a delay of two samples results in the bandpass filter response shown in Figure 5, illustrating the ripple in the pass-band and stop-band of the filter. Replacing the unit delay with a delay of 32 samples results in the filter response shown in Figure 6, which now has a set of 16 peaks and valleys uniformly spaced in frequency from 0 to $10 \mathrm{kHz}$. The filter coefficients are given in $\mathbf{A p p e n d i x ~} \mathbf{D}$; the comb filter has a total length of 961 taps, but is still computationally efficient since only 17 of these taps are non-zero.

The distortion measurement procedure is based on determining how much energy from the peaks of the combfiltered shaped noise signal spills over into the valleys. A total of 40,960 samples $(2.05 \mathrm{~s})$ of the hearing aid response to the test signal are acquired. As for the frequency-response measurements, and the first 4,096 samples (205 ms) are again discarded since they may be contaminated by transients. The power spectrum is computed using the method of modified periodigrams (21), with the data processed in blocks of 2,048 samples (102 ms) using a Hanning window and 50 percent overlap. The block length and type of window are important, since it is critical that leakage from the peaks of the spectrum not bias the estimated distortion levels in the valleys of the test signal. The distortion energy is computed as the mean-squared energy in the center of the valley, and the signal energy is computed as the mean-squared energy in the peaks to either side of the valley with the bias equal to the distortion energy subtracted out. The transition regions between the peaks and the valley are not used. The signal-to-distortion ratio (SDR) is then the square root of the ratio of the computed distortion energy to the signal energy, expressed as percent or in $\mathrm{dB}$
Table 1.

Weights for combining the signal-to-distortion ratios into a single distortion index.

\begin{tabular}{cccc}
\hline Band & $\begin{array}{c}\text { Valley Center } \\
\text { Frequency, } \mathbf{H z}\end{array}$ & $\begin{array}{c}\text { Band Edges, } \\
\mathbf{H z}\end{array}$ & Weight \\
\hline 1. & 625 & $312.5-937.5$ & 0.258 \\
2. & 1250 & $937.5-1562.5$ & 0.209 \\
3. & 1875 & $1562.5-2187.5$ & 0.180 \\
4. & 2500 & $2187.5-2812.5$ & 0.121 \\
5. & 3125 & $2812.5-3437.5$ & 0.084 \\
6. & 3750 & $3437.5-4062.5$ & 0.063 \\
7. & 4375 & $4062.5-4687.5$ & 0.047 \\
8. & 5000 & $4687.5-5312.5$ & 0.038 \\
\hline
\end{tabular}

for each valley frequency.

In addition to expressing the distortion as a function of frequency, there is a need for a single figure of merit to indicate the overall effect of the distortion on intelligibility. The figure of merit used in the broadband distortion measurement is a weighted SDR, computed using a procedure similar to that of the Articulation Index $(10,15)$. The SDRs are computed in $\mathrm{dB}$ for each valley frequency. $\mathrm{A}$ ratio greater than $30 \mathrm{~dB}$ is set to $30 \mathrm{~dB}$, and a ratio less than $0 \mathrm{~dB}$ is set to $0 \mathrm{~dB}$. The ratios are then multiplied by the weighting factors given in Table $\mathbf{1}$, summed, and divided by 30 to get a number between 0 and 1 . The weights are based on the number of the equally important bands for speech intelligibility that are included in each of the distortion bands. The weights from French and Steinberg (10) are for nonsense syllables; modifications to the weights can be made for connected discourse (17), but the change in the weighted SDR is small.

The above distortion measurement procedure was chosen over the magnitude-squared coherence function $(3,7)$ because of bias effects that can occur in computing the coherence. The magnitude-squared coherence function is the magnitude-squared cross-spectral density of the input and output signals, normalized by the product of the spectral densities of each of the two signals. The function is computed in the frequency domain by dividing the data into segments and averaging the appropriate fast Fourier transform (FFT) products over the segments. The coherence is high if the phase differences between the output and input remain constant from segment to segment as would occur for an ideal linear system, and is low if the phase differences fluctuate as would occur for noise or uncorrelated distortion. 


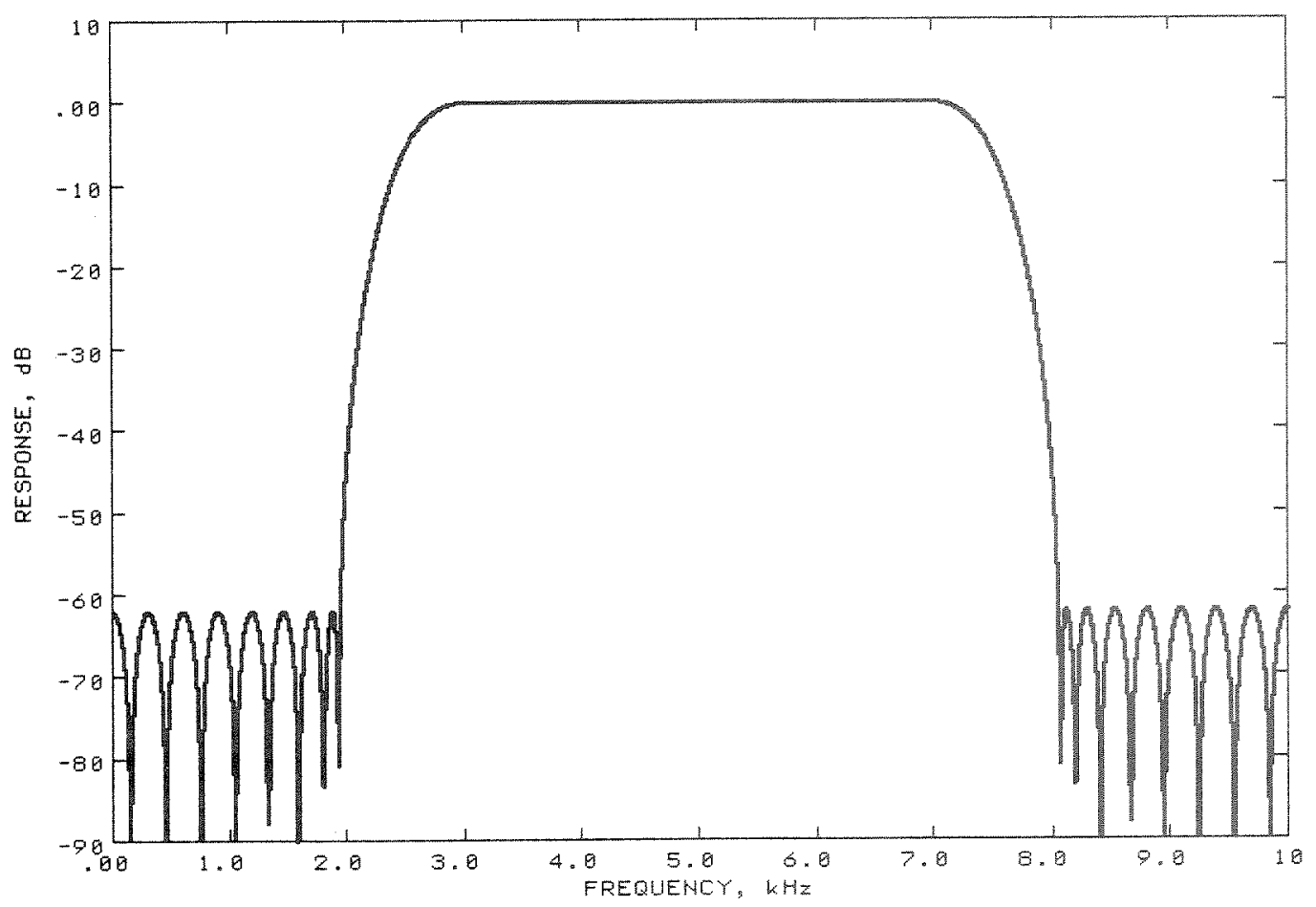

Figure 5.

Frequency response of the half-band filter having the unit delay replaced by a two-sample delay.

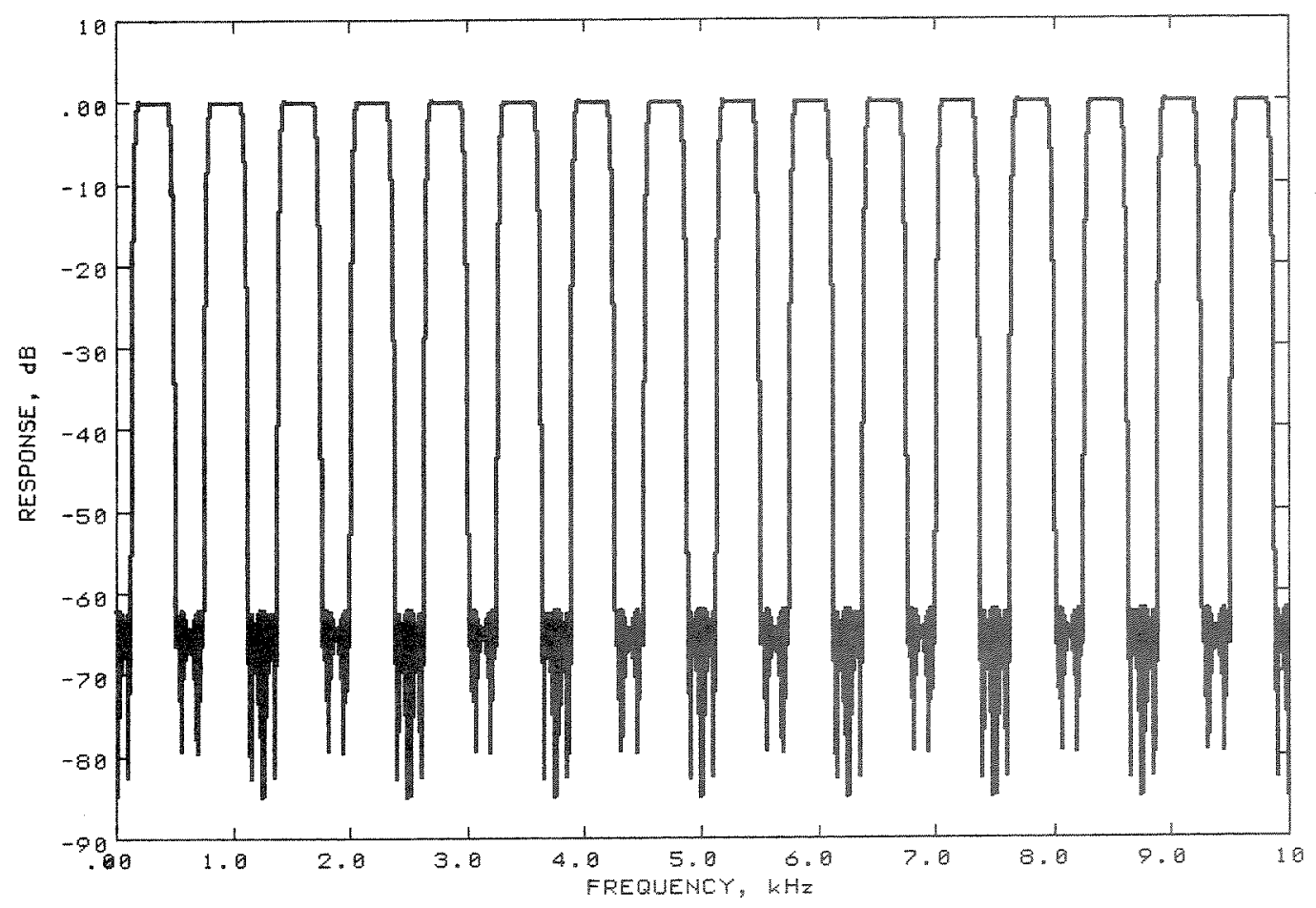

Figure 6.

Frequency response of the comb filter derived from the half-band filter by replacing the unit delay with a delay of 32 samples to give 16 combs. 
Journal of Rehabilitation Research and Development Vol. 27 No. 3 Summer 1990

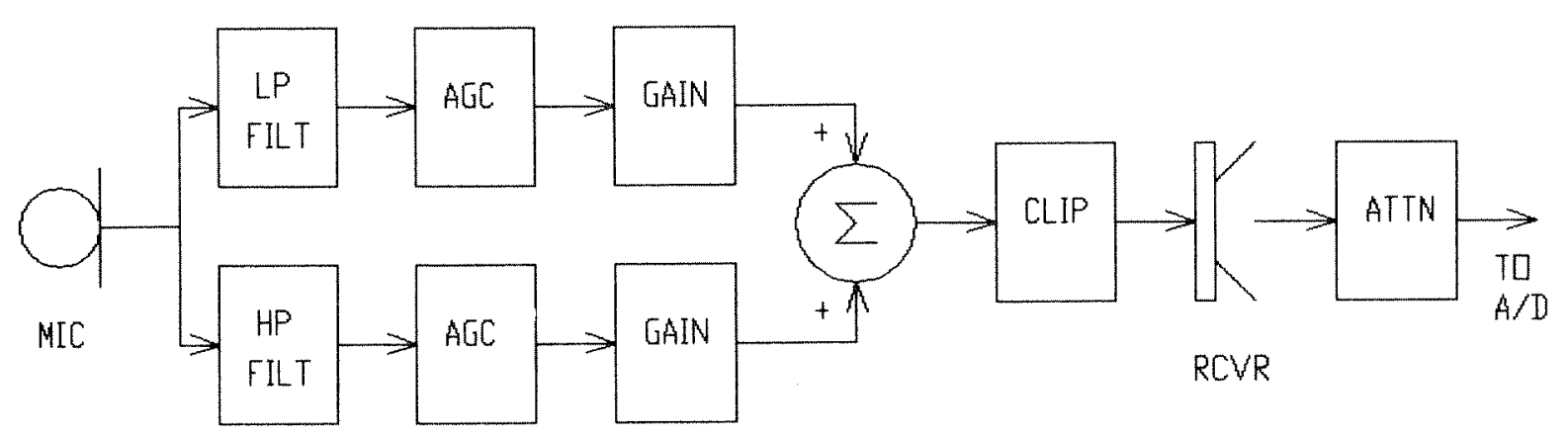

Figure 7.

Block diagram of the simulated hearing aid.

The bias in estimating the coherence function is inversely proportional to the number of data segmenpts used in its estimation $(7,8)$. This can be seen on an intuitive basis since the coherence function for a single segment is identically unity (no phase fluctuations can be detected if only one sample of the phase difference is available), so many segments will have to be processed in order to get an accurate estimate of a low coherence function value. Using small segments in order to reduce this bias, however, leads to a different set of problems, since misalignment of the time delays between the signals being cross-correlated leads to a bias that is inversely proportional to the segment length $(6,20)$. A related bias effect comes from the truncation of the system impulse response by the limited segment length. Examples comparing the distortion estimated using combfiltered noise and via the magnitude-squared coherence function are presented later in this paper.

\section{HEARING AID SIMULATIONS}

A digital simulation of a hearing aid is used to illustrate the test processing; the time-domain simulation is described by Kates (13) and a brief summary is given here. A block diagram of the simulated instrument is presented in Figure 7. The input to the microphone is the free-field sound pressure generated by an ideal loudspeaker. The test signal is the amplified 16-bit output of the computer $\mathrm{D} / \mathrm{A}$ converter. The microphone output is split into a low-pass and a high-pass channel, with independent input-referred compression (AGC-I) in each channel. The compression ratio and compression threshold can be specified for each channel. The gain in each channel is then adjusted, and the signals summed. The power amplifier is represented as a gain of $0 \mathrm{~dB}$ followed by symmetric output clipping. The receiver is assumed to be loaded acoustically by a 2 $\mathrm{cm}^{3}$ coupler; all other acoustic tubing and venting is ignored, so the output is representative of an in-the-ear (ITE) hearing aid as measured in a test chamber. The coupler output passes through an attenuator prior to being sampled by the 16-bit computer A/D converter. The computations for the simulation itself are all carried out using floating-point arithmetic.

Four simulated hearing aids are used to illustrate the test procedures. Two are linear instruments, and two are compression instruments. The linear hearing aid LOO is described by the parameters given in Table 2. This is a type of instrument commonly fitted for flat hearing losses. The two channels in this hearing aid have equal gains, and are equivalent to a single channel with a flat frequency response. Amplifier saturation is modeled by the clipping of all samples above a peak magnitude of $85 \mathrm{~dB}$ SPL less the microphone and channel gains. This distortion threshold is typical of commercially available hearing aids, and the symmetrical clipping is a reasonable model of amplifier saturation (13).

The second linear hearing aid L20 is identical to the first except that the low-frequency gain is set to $-20 \mathrm{~dB}$. This type of frequency response is commonly fitted for sloping hearing losses. The two linear channels are equivalent to a single-channel hearing aid with frequency shaping in the microphone response or in a preamplifier stage prior to the final amplifier. Again, amplifier saturation is modeled by the clipping of all samples above a peak magnitude of $85 \mathrm{~dB}$ less the microphone and channel gains.

The second pair of simulated hearing aids are twochannel compression instruments. Hearing aid $\mathrm{COO}$ is given by the parameters of Table 3. This hearing aid is typical of the newest products being introduced by the industry. The crossover frequency and the specified compression ratios and thresholds in each channel are meant for illustrative purposes only and do not necessarily represent a recommended set of parameters for an actual fitting. The 
two channels have equal gains, and are therefore typical of an instrument that would be fitted for a flat loss. The final amplifier stage and receiver in the simulated compression hearing aid are assumed to be the same as in the linear instrument, and the same clipping threshold for the instrument LOO is used.

The second compression hearing aid C20 is identical to the first except that the low-frequency gain is set to -20 $\mathrm{dB}$. This simulated instrument has a linear (low-level) frequency response typical to that fitted for a sloping hearing loss. All other parameters are the same as for hearing aid $\mathrm{C} 00$.

\section{EXAMPLES AND DISCUSSION}

\section{Frequency response}

The frequency-response test produces a family of response curves for shaped-noise input signals ranging from $60 \mathrm{~dB}$ SPL to $90 \mathrm{~dB}$ SPL in steps of $10 \mathrm{~dB}$. The sets of offset gain curves for the four simulated hearing aids are presented in Figure 8. The linear behavior of instrument L00 is shown by the uniform $10 \mathrm{~dB}$ separation between the lower three curves in Figure 8a, and the saturation of the amplifier becomes apparent for the $90 \mathrm{~dB}$ SPL input level since the last increment of $10 \mathrm{~dB}$ in the input results in about a $3 \mathrm{~dB}$ increase in the output at all frequencies. The linear hearing aid L20 shown in Figure $8 \mathbf{b}$ also exhibits the effects of saturation at the $90 \mathrm{~dB}$ SPL input level, but the effects are slightly less pronounced since the $-20 \mathrm{~dB}$ gain in the low-frequency channel results in less power

Table 2.

Parameters for the simulated linear hearing aid LOO.

Microphone:

Receiver:

Amplifier type:

Amplifier clipping level:

Crossover frequency:

$\begin{array}{ll}\text { Low-frequency channel } & \\ \text { Gain: } & 0 \mathrm{~dB} \\ \text { Compression ratio: } & 1: 1 \\ \text { Compression threshold: } & \text { Does not apply } \\ \text { Attack time: } & \text { Does not apply } \\ \text { Release time: } & \text { Does not apply } \\ \text { High-frequency channel } & \\ \text { Gain: } & 0 \mathrm{~dB} \\ \text { Compression ratio: } & 1: 1 \\ \text { Compression threshold: } & \text { Does not apply } \\ \text { Attack time: } & \text { Does not apply } \\ \text { Release time: } & \text { Does not apply }\end{array}$

Table 3.

Parameters for the simulated compression hearing aid $\mathrm{COO}$.

$\begin{array}{ll}\text { Microphone: } & \text { Knowles EA-1842 } \\ \text { Receiver: } & \text { Knowles ED-1913 } \\ \text { Amplifier type: } & \text { Current source } \\ \text { Amplifier clipping level: } & 85 \mathrm{~dB} \mathrm{SPL} \\ \text { Crossover frequency: } & 2 \mathrm{kHz} \\ \text { Low-frequency channel } & \\ \quad \text { Gain: } & 0 \mathrm{~dB} \\ \text { Compression ratio: } & 4: 1 \\ \text { Compression threshold: } & 75 \mathrm{~dB} \mathrm{SPL} \\ \text { Attack time: } & 1 \mathrm{msec} \\ \text { Release time: } & 50 \mathrm{msec} \\ \text { High-frequency channel } & \\ \text { Gain: } & 0 \mathrm{~dB} \\ \text { Compression ratio: } & 2: 1 \\ \text { Compression threshold: } & 65 \mathrm{~dB} \mathrm{SPL} \\ \text { Attack time: } & 1 \mathrm{msec} \\ \text { Release time: } & 50 \mathrm{msec}\end{array}$

reaching the amplifier for the same input stimulus level.

The families of frequency-response curves for the simulated compression instruments $\mathrm{C} 00$ and $\mathrm{C} 20$ are shown in Figures $\mathbf{8 c}$ and $\mathbf{8 d}$, respectively. At low frequencies, one can see the linear increase in gain for the input signal below the compression threshold of $75 \mathrm{~dB}$ SPL, followed by the closer spacing of the output curves as the input increases in level due to the 4:1 compression that is engaged for signals above threshold. At frequencies above the $2 \mathrm{kHz}$ crossover, the spacing of the curves reflects the lower threshold and 2:1 compression ratio chosen for the highfrequency channel. The combination of compression thresholds and compression ratios chosen for the two channels results in the response curve for the $90 \mathrm{~dB}$ SPL stimulus level being parallel to the response curve for the $60 \mathrm{~dB}$ SPL stimulus level for both simulated compression hearing aids. The measurements thus give an accurate indication of the frequency-response behavior of the hearing aid.

\section{Processing type}

The test to determine the type of processing and the number of bands produces a system identification pattern giving the response of the instrument under test as function of the sweep frequency. The patterns produced by the test program are quantized in level, with a gain reduction of less than $3 \mathrm{~dB}$ indicated by a dot, and a gain reduction of greater than $3 \mathrm{~dB}$ indicated by the symbol "\#." The resultant pattern is a $13 \times 13$ grid, since the adaptive filter coefficients are sampled each time the sweep frequency crosses the edge of one of the third-octave bands and the 

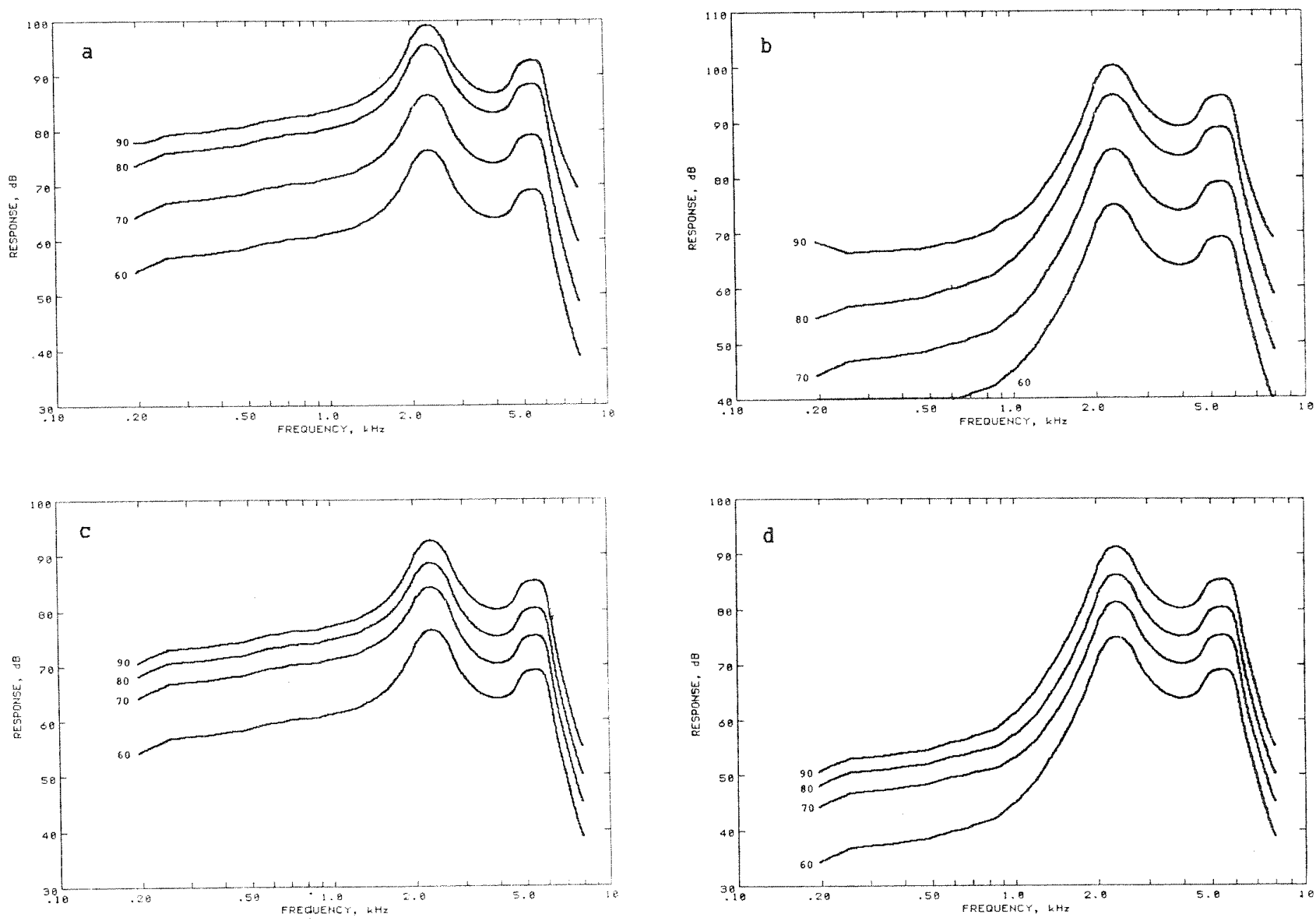

Figure 8.

Set of offset gain curves for input levels from $60 \mathrm{~dB}$ SPL to $90 \mathrm{~dB}$ SPL in steps of $10 \mathrm{~dB}$ for: a) the simulated linear hearing aid LO0; b) the simulated linear hearing aid $\mathrm{L} 20$; c) the simulated compression aid $\mathrm{CO0}$; and, d) the simulated compression aid $\mathrm{C} 20$.

filter frequency-response values are calculated for frequencies that are also at the edge of each of the 12 third-octave bands. The length of the adaptive filters used for the processing was set to 15 taps.

The system identification pattern for the simulated linear hearing aid LOO is shown in Figure 9a. The linear hearing aid processing results in essentially no gain reduction due to the swept bias tone. The one exception to this statement occurs at the top of the pattern, where the bias tone is amplified slightly by the $5 \mathrm{kHz}$ resonance peak of the microphone response and this causes some clipping in the amplifier. This clipping results in the apparent gain reduction at all frequencies shown in Figure 9 . The pattern match scores show a maximum for AGC processing, even though this is nominally a linear hearing aid, and indicate that the gain reduction occurs when the swept tone goes above $3150 \mathrm{~Hz}$.
The system identification pattern for the linear hearing aid L20 is shown in Figure $9 \mathrm{~b}$. The pattern is similar to that for the flat linear-response instrument, except that the amplifier saturation only reduces the gain in the vicinity of $2 \mathrm{kHz}$. The highest pattern match score is now for linear processing. Thus, changing the frequency response can affect the classification of a nominal linear instrument by changing the saturation effects.

The processing built into the simulated compression hearing aid $\mathrm{COO}$ is analyzed in Figure 9c. One can see the extent of the low-frequency channel, because there is a gain reduction everywhere in the channel for as long as the sweep is within it. As the sweep moves through the crossover region of the instrument, there is a gain reduction in both channels, and finally the high-frequency channel alone is affected as the sweep moves higher in frequency. Two-band compression has the highest processing score, 

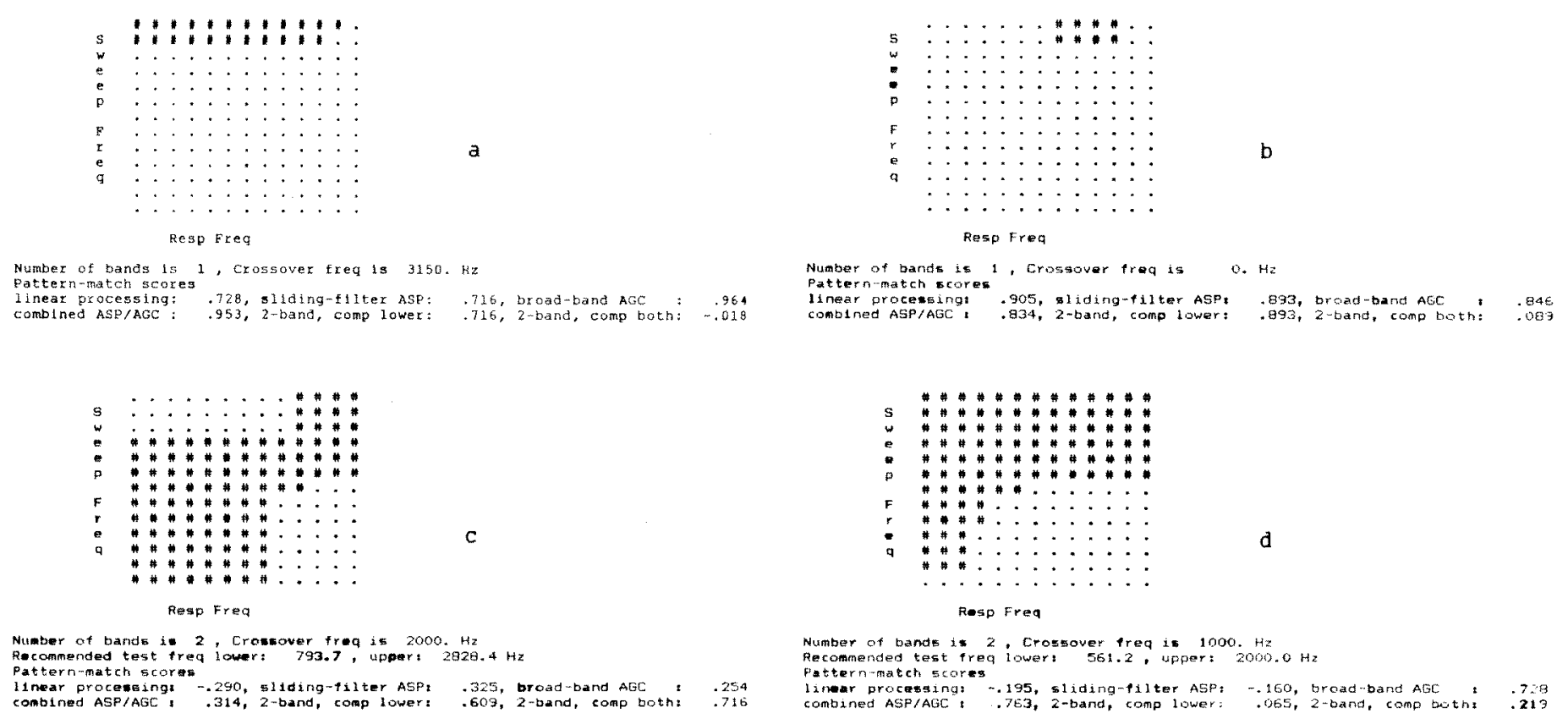

Figure 9.

System identification pattern and scores for: a) the simulated linear hearing aid L00; b) the simulated linear hearing aid L20; c) the simulated compression aid $\mathrm{C} 00$; and, d) the simulated compression aid $\mathrm{C} 20$.

and the program has correctly identified the $2 \mathrm{kHz}$ crossover frequency as well as the number of processing bands.

The pattern for the simulated compression hearing aid C20 is shown in Figure 9d. The pattern shows that when the sweep frequency is within the low-frequency channel, there is a gain reduction. The reduced gain, however, only appears in the lower half of the low-frequency channel. When the sweep is within the high-frequency channel, there appears to be a gain reduction at all frequencies rather than just in the high-frequency channel. The pattern matching scores reflect this behavior, since the broadband AGC and ASP/AGC both give higher scores than two-channel compression. The test pattern is a consequence of the highfrequency channel being given $20 \mathrm{~dB}$ more gain in the simulated instrument than the low-frequency channel. Because of this gain differential, the high-frequency channel dominates the frequency response of the instrument and the gain changes in the low-frequency channel have only a limited effect. Thus a two-channel compression instrument behaves more and more like a single-channel AGC instrument as the gain in one channel is increased relative to the gain in the other.

\section{Input/output characteristics}

The input/output characteristics for a linear hearing aid are determined at the ANSI (1) test frequency of $2 \mathrm{kHz}$.
The characteristics for a two-channel hearing aid are determined at the geometric center frequencies of each of the frequency bands found by the processing test. The crossover frequency was found to be $2 \mathrm{kHz}$ for the two-channel compression instrument $\mathrm{C} 00$. The closest third-octave test frequency for the low-frequency band is $800 \mathrm{~Hz}$, and for the high-frequency band, $3150 \mathrm{~Hz}$.

The input/output characteristics for the linear hearing aid LOO are given in Table 4. The linear operation is clearly indicated by the compression ratio of 1.0 for inputs up to $85 \mathrm{~dB}$ SPL. At higher stimulus intensities the amplifier clipping becomes increasingly more prominent, and the output saturation is reflected in the indicated high compression ratios. The behavior of the linear instrument L20 is similar, as shown by the data of Table $\mathbf{5}$, although the clipping is not quite as pronounced due to the $-20 \mathrm{~dB}$ lowfrequency gain of this simulated hearing aid.

The input/output characteristics for the compression hearing aid $\mathrm{COO}$ are presented in Table 6 for the test signals at $800 \mathrm{~Hz}$ and $3150 \mathrm{~Hz}$. With equal $0 \mathrm{~dB}$ gains for the two channels of the compression instrument, the low-frequency channel measurement comes close to showing the $4: 1 \mathrm{com}-$ pression ratio starting at a threshold of $75 \mathrm{~dB}$ SPL specified for this simulation. The high-frequency channel shows some minor influence from the low-frequency channel as its compression is engaged, but the measured compression 
Joumal of Rehabilitation Research and Development Vol. 27 No. 3 Summer 1990

Table 4.

Input/output measurements for the simulated linear hearing aid LOO.

\begin{tabular}{ccr}
\hline $\begin{array}{c}\text { Input } \\
\text { dB SPL }\end{array}$ & $\begin{array}{c}\text { Output } \\
\text { dBB SPL }\end{array}$ & $\begin{array}{r}\text { Freq }=2000 \mathrm{~Hz} \\
\text { Ratio }\end{array}$ \\
\hline 40 & 52.01 & - \\
45 & 56.96 & 1.01 \\
50 & 61.93 & 1.01 \\
55 & 66.97 & 0.99 \\
60 & 71.99 & 0.99 \\
65 & 77.00 & 1.00 \\
70 & 82.00 & 1.00 \\
75 & 86.99 & 1.00 \\
80 & 91.99 & 1.00 \\
85 & 94.11 & 2.36 \\
90 & 94.66 & 9.15 \\
95 & 94.93 & 18.04 \\
\hline
\end{tabular}

ratio is still reasonably close to the specified value of $2: 1$.

The input/output characteristics at the same pair of test frequencies for the simulated two-channel compression hearing aid $\mathrm{C} 20$ are given in Table 7. The compression ratio of 1.40 for the $45 \mathrm{~dB}$ SPL input signal level is an artifact caused by the A/D quantization of the low hearing aid output level since the low-frequency gain is -20 $\mathrm{dB}$. The compression ratio then becomes nearly unity over the linear region of the instrument, and becomes greater than one for input signal levels above the compression threshold. The compression ratio is not the $4: 1$ set in the parameters for the compression hearing aid, but rather a lower value because of the strong influence of the highfrequency channel due to its $20 \mathrm{~dB}$ greater gain. The input/output characteristics for the high-frequency channel tested at $3150 \mathrm{~Hz}$ very clearly show the compression threshold and 2:1 compression ratio set for the simulation because the reduced gain in the low-frequency channel minimizes its influence on the measured characteristics of the high-frequency channel.

The clearest indication of the behavior of an individual compression channel can be found when the other channel is turned off. Otherwise the measured input/output characteristics will tend to reflect the dominant frequency band no matter which channel is being measured. As the gain in one channel is increased relative to the other, the
Table 5.

Input/output measurements for the simulated linear hearing aid L20.

\begin{tabular}{ccc}
\hline $\begin{array}{c}\text { Input } \\
\text { dB SPL }\end{array}$ & $\begin{array}{c}\text { Output } \\
\text { dB SPL }\end{array}$ & $\begin{array}{c}\text { Comp } \\
\text { Ratio }\end{array}$ \\
\hline 40 & 48.93 & - \\
45 & 54.00 & 0.99 \\
50 & 58.94 & 1.01 \\
55 & 64.03 & 0.98 \\
60 & 69.03 & 1.00 \\
65 & 74.03 & 1.00 \\
70 & 79.03 & 1.00 \\
75 & 84.03 & 1.00 \\
80 & 89.02 & 1.00 \\
85 & 93.52 & 1.11 \\
90 & 94.32 & 6.20 \\
95 & 94.90 & 8.63 \\
\hline
\end{tabular}

hearing aid behaves more and more like a single-channel instrument. Thus the relative gains in the different frequency channels can be an important factor in the measured compression ratios and thresholds.

\section{Attack and release times}

The measured attack and release times depend on both the system under test and the measurement procedure. The presence of the all-pass filter shown in the envelope detection system of Figure 4 will affect the estimated attack and release times due to the transient behavior of the analysis filter itself. Additional transient effects come from the inherent linear behavior of the hearing aid, particularly from the receiver response. The degree of overshoot for the computed envelope of the output of the linear hearing aid L00, excited by the stepped $2 \mathrm{kHz}$ excitation, is shown in Figure 10a. The behavior of linear instrument L20 in Figure $10 \mathrm{~b}$ is quite similar. There is less than $2 \mathrm{~dB}$ of overshoot on the attack portion of the signal, and less than $2 \mathrm{~dB}$ of undershoot on the release portion. This is below the criterion for the ANSI S3.22 test procedure, so the reported attack and release times are both zero. Neither the envelope computation nor the behavior of the linear elements of the hearing aid will significantly bias the calculation of the attack and release times.

The envelopes are computed at both test frequencies 
Table 6.

Input/output measurements for the simulated compression hearing aid $\mathrm{COO}$.

\begin{tabular}{ccccc}
\hline & \multicolumn{2}{c}{ Freq } & 800 Hz & \multicolumn{2}{c}{ Freq $=3150 \mathrm{~Hz}$} \\
Inpur & Output & Comp & Output & Comp \\
diB SPL & dBS SPL & Ratio & dB SPL & Ratio \\
\hline 40 & 40.33 & - & 45.98 & - \\
45 & 45.20 & 1.03 & 50.96 & 1.01 \\
50 & 50.21 & 1.00 & 55.95 & 1.00 \\
55 & 55.21 & 1.00 & 60.96 & 1.00 \\
60 & 60.22 & 1.00 & 65.67 & 1.06 \\
65 & 65.21 & 1.00 & 68.33 & 1.88 \\
70 & 70.22 & 1.00 & 71.10 & 1.80 \\
75 & 73.70 & 1.43 & 74.05 & 1.70 \\
80 & 74.98 & 3.91 & 77.25 & 1.56 \\
85 & 76.30 & 3.80 & 80.00 & 1.82 \\
90 & 77.64 & 3.72 & 82.07 & 2.41 \\
95 & 78.96 & 3.80 & 84.22 & 2.33 \\
\hline
\end{tabular}

for the two simulated two-channel compression hearing aids. The envelope for the $800 \mathrm{~Hz}$ test frequency, which excites primarily the low-frequency channel, is shown in Figure $10 \mathrm{c}$ for instrument $\mathrm{COO}$, and in Figure $10 \mathrm{e}$ for instrument $\mathrm{C} 20$. The measured attack times are $2.6 \mathrm{~ms}$ for $\mathrm{C} 00$ and $1.4 \mathrm{~ms}$ for $\mathrm{C} 20$. These values are reasonably close to the specified time of $1 \mathrm{~ms}$, but the measured release times of 10.3 and $12.7 \mathrm{~ms}$ for $\mathrm{C} 00$ and $\mathrm{C} 20$, respectively, are much shorter than the specified release time of $50 \mathrm{~ms}$.

The difference between the estimated and specified release times is a result of the compression threshold of $75 \mathrm{~dB}$ SPL set in the low-frequency channel. This threshold is close to the upper signal level of $80 \mathrm{~dB}$ SPL. After the test signal returns to the $55 \mathrm{~dB}$ SPL level, the hearing aid spends only a short period of time in compression before the detection circuit indicates that the gain should return to the linear value used for inputs below the compression threshold. Thus the measured release time is a function of the compression threshold; the higher the threshold, the shorter the apparent release time, all else being held constant.

The test signal at $3150 \mathrm{~Hz}$ excites primarily the highfrequency channel. The envelopes for simulated compression instruments $\mathrm{COO}$ and $\mathrm{C} 20$ are shown in Figures $10 \mathrm{~d}$ and 10f, respectively. The measured attack times are 1.65 $\mathrm{ms}$ for instrument $\mathrm{COO}$ and $1.75 \mathrm{~ms}$ for $\mathrm{C} 20$, and the
Table 7.

Input/output measurements for the simulated compression hearing aid $\mathrm{C} 20$.

\begin{tabular}{|c|c|c|c|c|}
\hline \multirow[b]{2}{*}{$\begin{array}{l}\text { Input } \\
\text { dP SPL }\end{array}$} & \multicolumn{2}{|c|}{ Freq $=800 \mathrm{~Hz}$} & \multicolumn{2}{|c|}{ Freq $=3150 \mathrm{~Hz}$} \\
\hline & $\begin{array}{l}\text { Output } \\
\text { dP SPL }\end{array}$ & $\begin{array}{l}\text { Comp } \\
\text { Ratio }\end{array}$ & $\begin{array}{l}\text { Ontpue } \\
\text { aB S SPL }\end{array}$ & $\begin{array}{l}\text { Comp } \\
\text { Patio }\end{array}$ \\
\hline 40 & 23.27 & - & 45.77 & - \\
\hline 45 & 26.85 & 1.40 & 50.77 & 1.00 \\
\hline 50 & 31.75 & 1.02 & 55.75 & 1.00 \\
\hline 55 & 36.43 & 1.07 & 60.76 & 1.00 \\
\hline 60 & 41.52 & 0.98 & 65.45 & 1.07 \\
\hline 65 & 46.54 & 1.00 & 67.95 & 2.00 \\
\hline 70 & 51.49 & 1.01 & 70.46 & 2.00 \\
\hline 75 & 55.41 & 1.28 & 72.96 & 1.99 \\
\hline 80 & 58.28 & 1.74 & 75.47 & 1.99 \\
\hline 85 & 61.94 & 1.37 & 77.98 & 2.00 \\
\hline 90 & 65.19 & 1.54 & 80.47 & 2.01 \\
\hline 95 & 67.49 & 2.17 & 82.97 & 2.00 \\
\hline
\end{tabular}

measured release times are $53.8 \mathrm{~ms}$ for $\mathrm{COO}$ and $50.9 \mathrm{~ms}$ for $\mathrm{C} 20$, respectively. These measured values are close to the specified attack time of $1 \mathrm{~ms}$ and release time of 50 $\mathrm{ms}$. The longer release times are due to the lower compression threshold in the high-frequency channel. However, the effect of the compression threshold on the attack times appears to be much smaller.

\section{Broadband distortion}

The broadband distortion measurement determines the amount of energy in the valleys of the comb-filtered shaped noise signal relative to the energy in the two adjacent peaks. The detection threshold depends on the quantization noise in the computer $\mathrm{D} / \mathrm{A}$ and $\mathrm{A} / \mathrm{D}$ converters as well as the side-lobe levels of the comb filter and the round-off error and leakage in the discreet Fourier transform used for the spectral analysis. The internal noise floor of a typical hearing aid, not included in the simulation, will also affect the measured distortion levels, because the measurement procedure cannot distinguish between distortion and noise. The proper levels for the test signal represent a trade-off between the desire on the one hand for a high signal level to minimize the relative importance of the internal and measurement noise, and the desire on the other hand for a low signal level to insure at least one set of measurements in the linear operating range of the hearing aid. 
Journal of Rehabilitation Research and Development Vol. 27 No. 3 Summer 1990
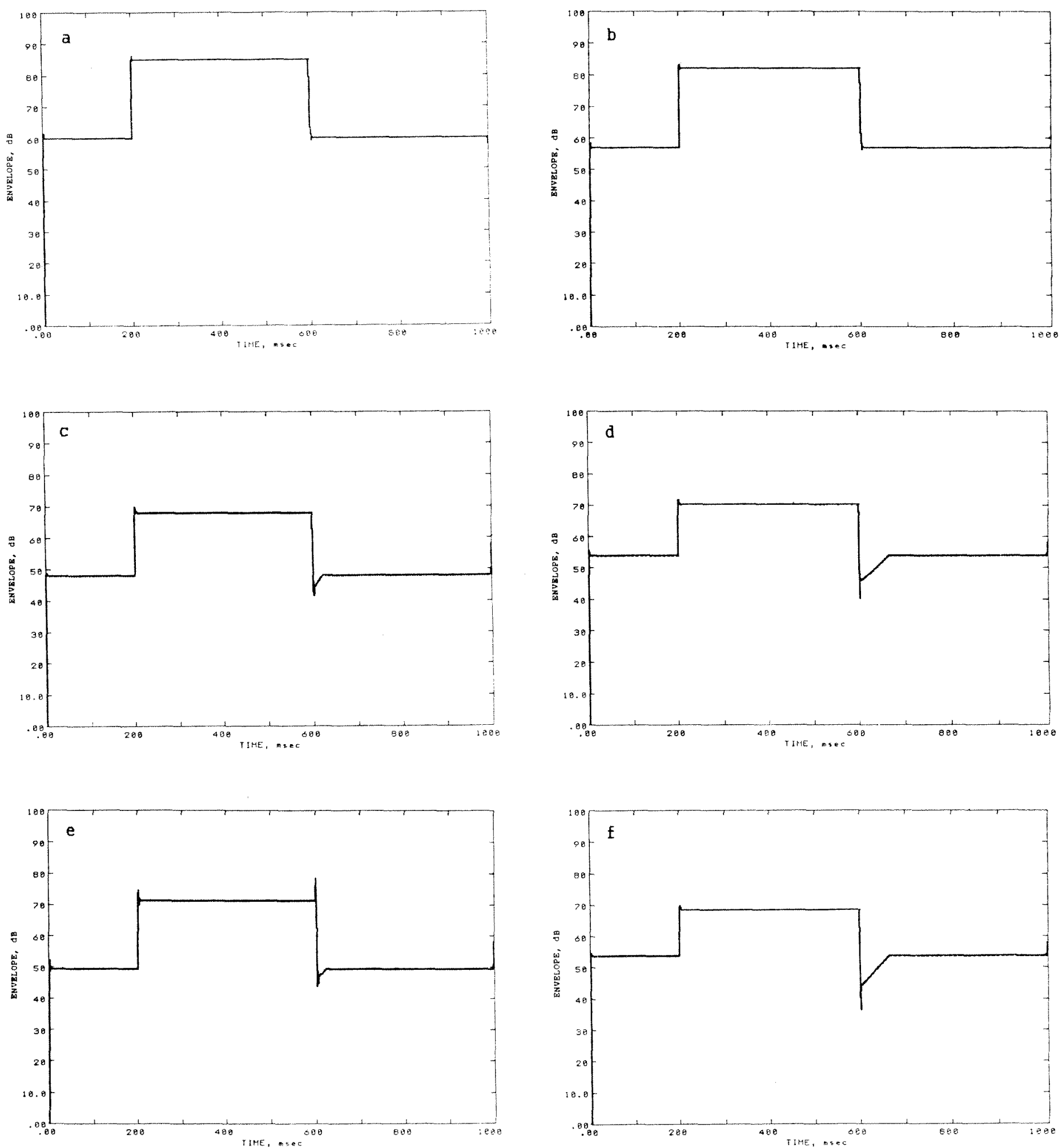

Figure 10.

Signal envelope from the attack and release time measurements of: a) the simulated linear hearing aid L00 measured at $2000 \mathrm{~Hz}$; b) the simulated linear hearing aid L20 measured at $2000 \mathrm{~Hz}$; c) the simulated compression aid C00 measured at $800 \mathrm{~Hz}$; d) the simulated compression aid $\mathrm{C} 00$ measured at $3150 \mathrm{~Hz}$; e) the simulated compression aid $\mathrm{C} 20$ measured at $800 \mathrm{~Hz}$; and, f) the simulated compression aid C20 measured at $3150 \mathrm{~Hz}$. 
A set of broadband distortion measurements for the simulated linear hearing aid L00 is presented in Table 8, and the corresponding data for the linear instrument L20 is presented in Table 9. The percent distortion in the valleys from 625 through $5000 \mathrm{~Hz}$ are given for input signal levels from 60 through $90 \mathrm{~dB}$ SPL, along with the weighted SDR index. The lowest distortion values are for the input signal at $70 \mathrm{~dB}$ SPL, not at $60 \mathrm{~dB}$ SPL; both signal levels are well below the amplifier clipping threshold, but the lower input level is affected more by the quantization noise of the digital sampling process. The response of linear instruments L00 and L20 to the $70 \mathrm{~dB}$ SPL input signal is shown in Figure 11a and Figure 11b, respectively, where the energy in the valleys falls below the frequency-response axis. The bulk of the measured values for the $70 \mathrm{~dB} \mathrm{SPL}$ input signal show that the procedure is capable of measuring distortion at the level of 0.1 percent when the signal level is carefully adjusted.

Increasing the level of the test signal above $70 \mathrm{~dB}$ SPL results in increased distortion. The data for the $80 \mathrm{~dB} \mathrm{SPL}$ test signal presented in Table 8 and Table 9 shows a substantial increase in the measured distortion when compared with the lower signal levels. Since the distortion levels are still relatively low, however, the weighted SDR index only shows a modest decrease to the value of 0.821 for instrument L00, and 0.951 for instrument L20.

Increasing the signal level still further to $90 \mathrm{~dB}$ SPL results in a large amount of clipping in the simulated amplifier. This in turn greatly increases the measured distortion. The distortion products tend to have a flat spec-

Table 8.

Ratio of distortion to signal level and the weighted SDR index as a function of the input signal intensity for the simulated linear hearing aid LOO.

\begin{tabular}{cllll}
\hline $\begin{array}{c}\text { Frequency, } \\
\mathbf{H z}\end{array}$ & \multicolumn{4}{c}{ Input Signal Intensity, $\mathbf{d B} \mathbf{S P L}$} \\
\hline 625 & $0.08 \%$ & $0.07 \%$ & $5.18 \%$ & $16.38 \%$ \\
1250 & 0.09 & 0.06 & 5.86 & 18.15 \\
1875 & 0.07 & 0.05 & 5.07 & 15.73 \\
2500 & 0.11 & 0.06 & 6.58 & 21.27 \\
3125 & 0.12 & 0.06 & 6.35 & 21.85 \\
3750 & 0.17 & 0.08 & 7.16 & 23.87 \\
4375 & 0.20 & 0.09 & 7.73 & 26.56 \\
5000 & 0.23 & 0.09 & 8.35 & 28.29 \\
Index & 1.000 & 1.000 & 0.821 & 0.484 \\
\hline
\end{tabular}

trum, which is then shaped by the receiver response. The spectrum of the distortion is thus flatter than the spectrum of the linear response of instrument L20 with its $20 \mathrm{~dB}$ lower gain at low frequencies; this results in a greater relative amount of distortion at low frequencies than at high frequencies for this simulated hearing aid. This is illustrated in Figures $\mathbf{1 2 a}$ and $\mathbf{1 2 b}$, which show the linear hearing aid output for the $90 \mathrm{~dB}$ SPL input. The distortion has filled in the valley at $625 \mathrm{~Hz}$ for instrument L20 to a much greater degree than the valleys above $2 \mathrm{kHz}$, while the flatter response of instrument $\mathrm{LOO}$ results in more uniform relative distortion amounts at all frequencies. The weighted SDR index for the $90 \mathrm{~dB}$ SPL test signal for instrument L20 has been reduced to 0.472 , and for instrument $L 00$ the value is 0.484 . One would expect some reduction in speech intelligibility under these circumstances.

The distortion measurements were then repeated for the simulated compression hearing aids, and the results are presented in Table $\mathbf{1 0}$ for instrument $\mathrm{C00}$, and in Table 11 for instrument C20. The results for the $60 \mathrm{~dB}$ SPL input level are very similar to those for the linear hearing aids because the signal is below the compression thresholds in both channels. Increasing the signal level to $70 \mathrm{~dB}$ SPL results in an increase in distortion because the rapid attack time of the compression processing distorts the peaks of the test signal. The distortion can also be seen in Figures 11c and 11d, where the valleys in the hearing aid output for the $70 \mathrm{~dB}$ SPL input contain more energy than those of Figures 11a and $\mathbf{1 1 b}$ for the simulated linear instruments. Increasing the test signal level to 80 and then $90 \mathrm{~dB}$ SPL,

Table 9.

Ratio of distortion to signal level and the weighted SDR index as a function of the input signal intensity for the simulated linear hearing aid L20.

\begin{tabular}{cllll}
\hline $\begin{array}{c}\text { Frequency, } \\
\mathbf{H z}\end{array}$ & \multicolumn{4}{c}{ Input Signal Intensity, $\mathbf{d B}$ SPL } \\
\hline 625 & $0.16 \%$ & $0.15 \%$ & $6.11 \%$ & $\mathbf{9 0}$ \\
1250 & 0.08 & 0.07 & 3.01 & 25.54 \\
1875 & 0.06 & 0.04 & 1.07 & 9.51 \\
2500 & 0.12 & 0.07 & 1.44 & 12.82 \\
3125 & 0.13 & 0.06 & 1.44 & 11.06 \\
3750 & 0.17 & 0.08 & 1.54 & 13.18 \\
4375 & 0.21 & 0.09 & 1.66 & 14.09 \\
5000 & 0.22 & 0.09 & 1.78 & 15.42 \\
Index & 1.000 & 1.000 & 0.951 & 0.472 \\
\hline
\end{tabular}



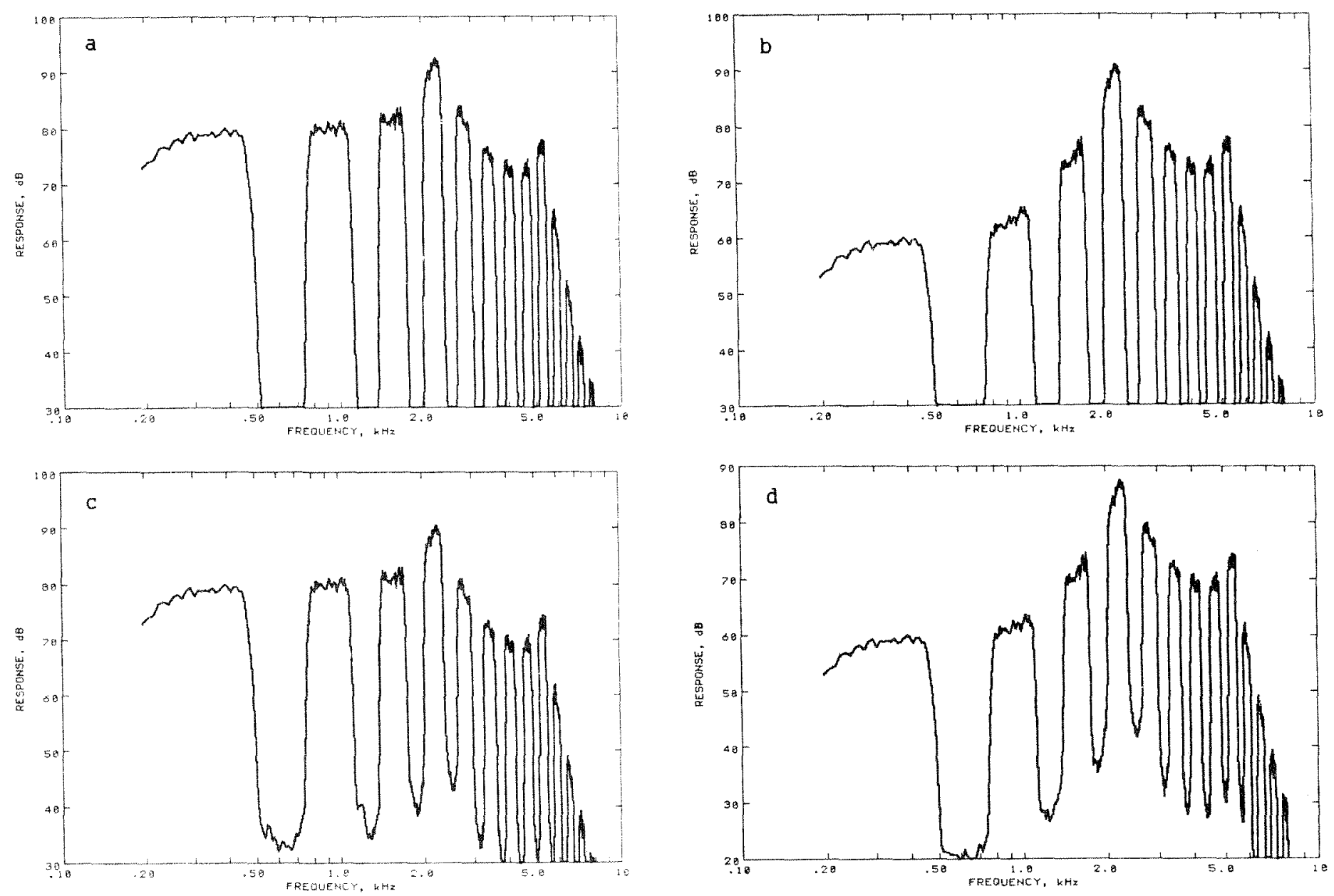

Figure 11.

Output spectrum for the comb-filtered shaped noise signal input at $70 \mathrm{~dB}$ SPL for: a) the simulated linear hearing aid L00; b) the simulated linear hearing aid $\mathrm{L} 20$; c) the simulated compression aid $\mathrm{C} 00$; and, d) the simulated compression aid $\mathrm{C} 20$.

however, results in only small increases in the distortion levels because the compression prevents the peaks from exceeding the clipping threshold of the amplifier. The small amount of distortion can also be seen in the compression hearing aid frequency-response curves of Figures $12 \mathrm{c}$ and $12 \mathrm{~d}$ for the $90 \mathrm{~dB}$ SPL input signal, where the energy in the valleys is not very different from the levels for the 70 $\mathrm{dB}$ SPL input as shown in Figure 11. Also note that the weighted SDR index for the compression instrument remains at 1.000 for all the test signal levels.

Distortion measurements using the coherence function

The SDR can also be derived from the magnitudesquared coherence function using the relationship:

$$
\operatorname{SDR}(f)=\frac{\gamma^{2}(f)}{1-\gamma^{2}(f)}
$$

where $\gamma^{2}(f)$ is the magnitude-squared coherence function. Because the coherence function is a two-channel measurement procedure, processing the same amount of data as for the comb-filtered noise procedure takes twice the number of FFTs per data segment and twice the amount of processing time. Plots of the SDR were derived from the coherence function and smoothed in overlapping thirdoctave bands for the shaped-noise input signal ranging in level from 60 to $90 \mathrm{~dB}$ SPL. The data were processed in segments of 2,048 samples (102 ms) using a Hanning window and 50 percent overlap just as was done for the comb-filtered noise signal.

The SDR derived from the coherence function are presented in Figures 13a and 13b for the linear instrument $\mathrm{L} 20$ and the compression instrument $\mathrm{C} 20$, respectively. The curves in Figure 13a for the 60 and $70 \mathrm{~dB}$ SPL input levels overlap for the linear hearing aid, and give a SDR of about 

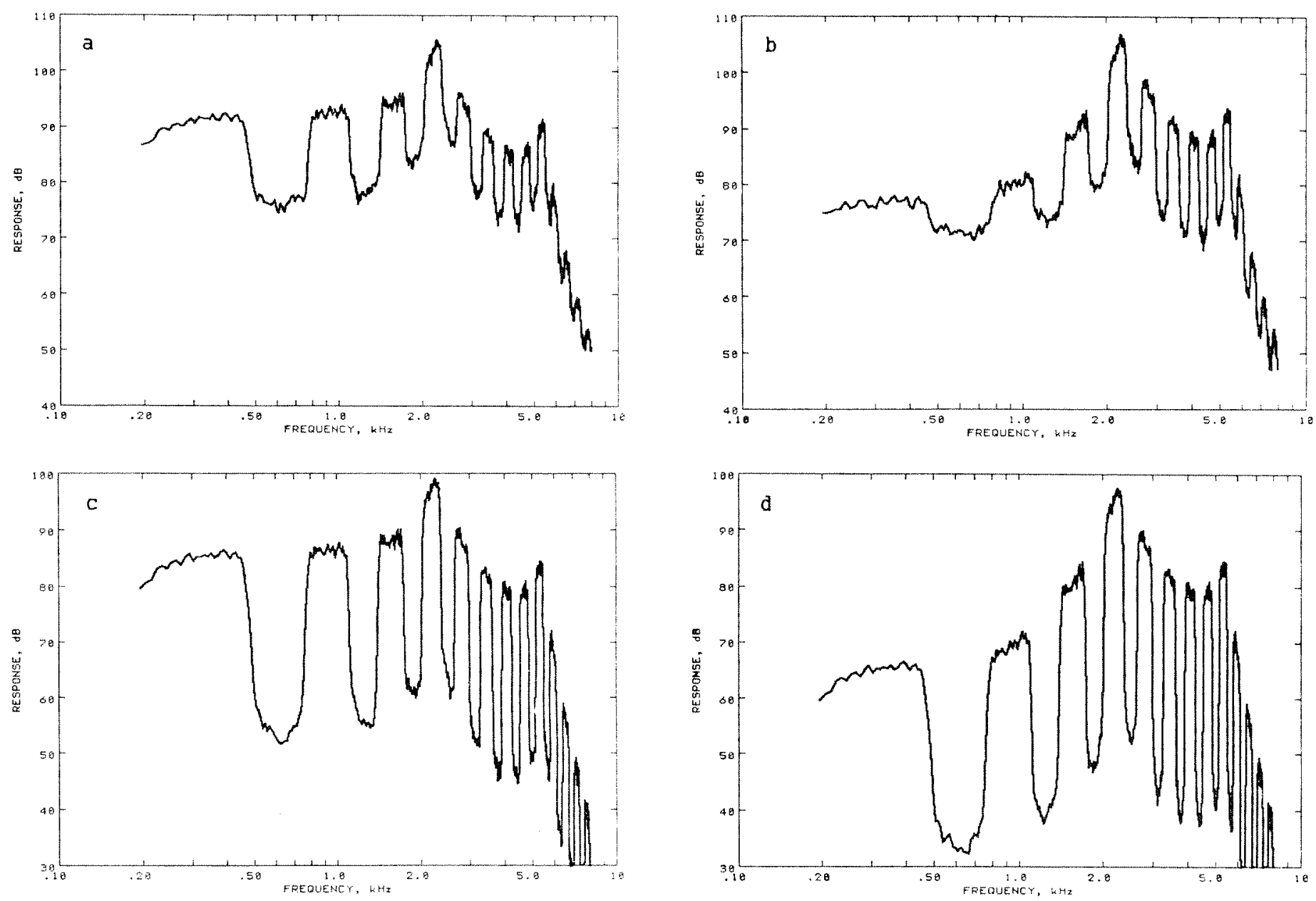

Figure 12.

Output spectrum for the comb-filtered shaped noise signal input at $90 \mathrm{~dB}$ SPL for: a) the simulated linear hearing aid L00; b) the simulated linear hearing aid $\mathrm{L} 20$; c) the simulated compression aid $\mathrm{C} 00$; and, d) the simulated compression aid $\mathrm{C} 20$.

$34 \mathrm{~dB}$ in the vicinity of $1 \mathrm{kHz}$. This should be compared with the distortion value of 0.08 percent measured at 1250 $\mathrm{Hz}$ for the same linear instrument L20 using the combfiltered noise signal, which corresponds to a SDR of about $62 \mathrm{~dB}$. The SDR curves in Figure 13a for the 80 and 90 dB SPL input levels lie lower than the SDR curves for the lower input levels, as would be expected, and again, the SDR values are much lower than for the comb-filtered noise. The set of SDR curves is repeated in Figure 13b for the simulated compression instrument $\mathrm{C} 20$. While the trend of the distortion behavior is similar to that found with the comb-filtered noise procedure, all of the curves underestimate the SDR as compared with the comb-filtered noise measurements.

The low estimated SDR from the coherence function is the result of the bias in the estimation procedure due to truncating the data segments. Doubling the length of the data segment to 4,096 samples $(205 \mathrm{~ms})$ increases the estimated SDR by $6 \mathrm{~dB}$, but also increases the variance since only half the number of data segments can be extracted from the same amount of test data. To increase the estimated SDR from 34 to $64 \mathrm{~dB}$, while keeping the variance of the estimate constant at each frequency before smoothing, would require increasing the data segment length by a factor of 32 , while keeping the number of segments the same. This would require processing 32 times the amount of data to achieve the same accuracy in the estimate as is achieved by testing with the comb-filtered noise signal. If third-octave smoothing is used, the number of data segments needed for the coherence function estimate can be reduced since the smoothing over adjacent frequency points reduces the variance, but it will still require larger FFTs and more processing time than for the comb-filtered noise procedure. 
Journal of Rehabilitation Research and Developmen Vol. 27 No. 3 Summer 1990

Table 10 .

Ratio of distortion to signal level and the weighted SDR index as a function of the input signal intensity for the simulated compression hearing aid $\mathrm{COO}$.

\begin{tabular}{ccccc}
\hline $\begin{array}{c}\text { Frequency, } \\
\mathrm{Hz}\end{array}$ & 60 & $\begin{array}{c}\text { Input Signal Intensity, dP SPL } \\
\text { 70 }\end{array}$ & 80 & 90 \\
\hline 625 & $0.08 \%$ & $0.53 \%$ & $2.01 \%$ & $2.38 \%$ \\
1250 & 0.09 & 0.70 & 2.21 & 2.72 \\
1875 & 0.07 & 0.55 & 2.04 & 2.28 \\
2500 & 0.12 & 0.79 & 1.87 & 2.53 \\
3125 & 0.13 & 0.89 & 1.54 & 2.30 \\
3750 & 0.17 & 0.96 & 1.28 & 2.25 \\
4375 & 0.20 & 1.03 & 1.16 & 2.36 \\
5000 & 0.23 & 1.03 & 1.13 & 2.45 \\
Index & 1.000 & 1.000 & 1.000 & 1.000 \\
\hline
\end{tabular}

Table 1 .

Ratio of distortion to signal level and the weighted SDR index as a function of the input signal intensity for the simulated compression hearing aid $\mathrm{C} 20$.

\begin{tabular}{ccccc} 
Frequency, & \multicolumn{5}{c}{ Input Signal } & Intensity, dB SPL \\
$\mathrm{H} z$ & 60 & 70 & 80 & 90 \\
\hline 625 & $0.09 \%$ & $1.03 \%$ & $2.08 \%$ & $2.15 \%$ \\
1250 & 0.07 & 1.17 & 1.47 & 1.40 \\
1875 & 0.06 & 0.71 & 0.79 & 0.76 \\
2500 & 0.13 & 0.93 & 0.95 & 0.95 \\
3125 & 0.13 & 0.89 & 0.90 & 0.90 \\
3750 & 0.17 & 0.92 & 0.92 & 0.92 \\
4375 & 0.20 & 1.01 & 1.01 & 1.02 \\
5000 & 0.23 & 1.01 & 1.01 & 1.01 \\
Index & 1.000 & 1.000 & 1.000 & 1.000 \\
\hline
\end{tabular}
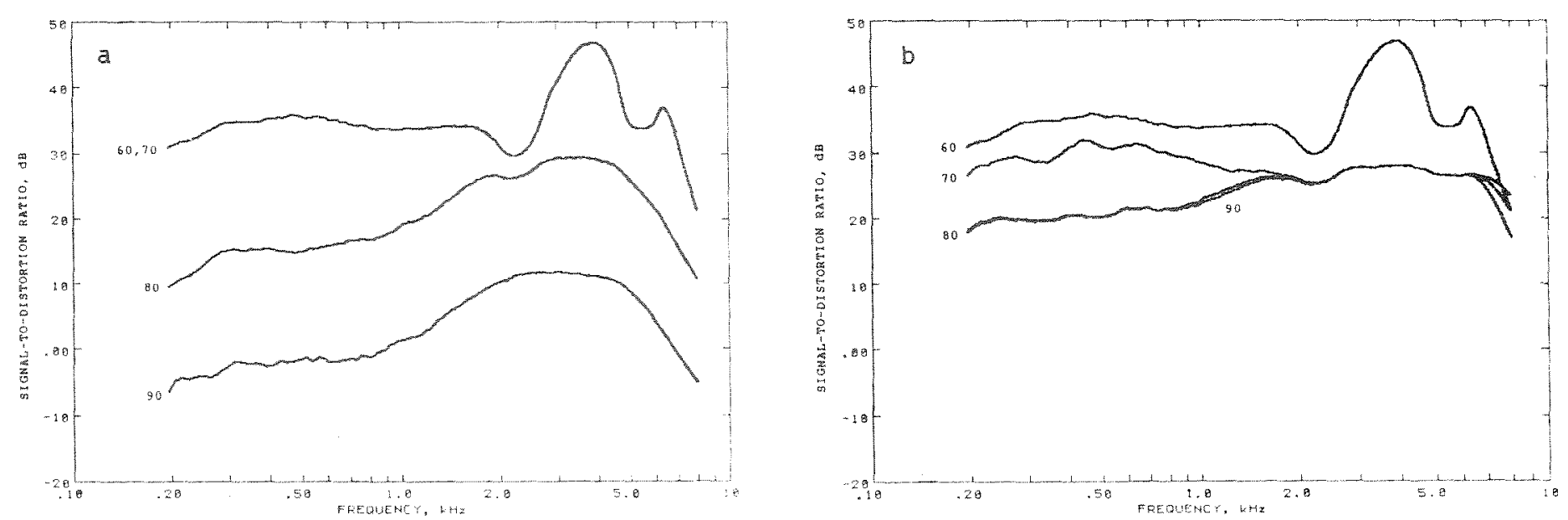

Figure 13.

Signal-to-distortion ratio derived from the magnitude-squared coherence function as the excitation goes from 60 to $90 \mathrm{~dB}$ SPL in steps of $10 \mathrm{~dB}$ for: a) the simulated linear hearing aid $\mathrm{L} 20$; and, b) the simulated compression hearing aid $\mathrm{C} 20$. 


\section{CONCLUSIONS}

This paper has presented an integrated test suite for evaluating an arbitrary commercially available hearing aid. The set of tests consists of the frequency response as a function of the test signal level, the processing type and number of processing bands, input/output characteristics for each identified processing band, attack and release times for each band, and frequency-dependent broadband distortion as a function of the input signal level along with an overall weighted signal-to-distortion index. Existing test procedures have been used for the frequency-response, input/output, and attack and release time measurements. New procedures have been derived for determining the processing type and number of bands, and for measuring the broadband distortion.

The test procedure for determining the number of processing bands currently tests for only one or two bands. The procedure can be extended to a greater number of bands by increasing the length of time for the sweep to traverse the frequency range, and by increasing the number of taps in the adaptive filters. This will increase the resolution of the procedure and improve the ability to identify the band edges, but at the price of increased processing time. The set of idealized test patterns can then be extended

\section{REFERENCES}

1. American National Standards Institute: American National Standard Specification of Hearing Aid Characteristics, ANSI S3.22-1982. New York: American National Standards Institute, 1982.

2. American National Standards Institute: American National Standard Specification for Octave, Half-Octave, and Third-Octave Band Filter Sets, ANSI S1.11-1966 (R 1976). New York: American National Standards Institute, 1976.

3. Benignus VA: Estimation of the coherence spectrum and its confidence interval using the fast Fourier transform. IEEE Trans Audio Electroacoust 17(2):145-150, 1969.

4. Burnett ED: A new method for the measurement of nonlinear distortion using a random noise test signal. Bull Prosthet Res 10(7):76-92, 1967.

5. Burnett ED, Bartel TW, Roland WR: NBS hearing aid test procedures and test data. In: Handbook of Hearing Aid Measurement 1987, IB 11-78 (revised), 9-30. Washington, DC: Veterans Administration, 1987. to include three or more channels of independent compression or any other processing or combinations of processing to be checked.

The test suite is suitable for the automated testing of a hearing aid. The number of processing bands and the band edges determine the frequencies to be used for testing the input/output characteristics and the attack and release times, so a simple algorithm can step through the five sets of tests presented in this paper in the order presented and produce a set of test results at the end.

The test suite has been designed for implementation on a personal computer containing a $A / D$ and $D / A$ converter board for the signal generation and data acquisition. Such a computer system is being designed at the Lexington Center for measuring actual hearing aids. Test results from this implementation will be available at a later date, and will allow the comparison of the test procedures presented in this paper with other measurement protocols.

\section{ACKNOWLEDGMENT}

The research presented in this paper was supported by Grant No. G00830251 from the National Institute on Disability and Rehabilitation Research.
6. Carter GC: Bias in magnitude-squared coherence estimation due to misalignment. IEEE Trans Acoust Speech Signal Process 28:97-99, 1980.

7. Carter GC, Knapp CH, Nuttall AH: Estimation of the magnitude-squared coherence function via overlapped fast Fourier transform processing. IEEE Trans Audio Electroacoust 21(4):337-344, 1973.

8. Carter GC, Knapp CH, Nuttall AH: Statistics of the estimate of the magnitude-coherence function. IEEE Trans Audio Electroacoust 21(4):388-389, 1973.

9. Chang CY: Adaptive multichannel filtering. Proc Int Conf Acoust Speech Signal Proc 462-465, 1980.

10. French $\mathbf{N R}$, Steinberg JC: Factors governing the intelligibility of speech sounds. I Acoust Soc Am 19:90-119, 1949.

11. Griffiths LJ: An adaptive lattice structure for noisecancelling applications. Proc Int Conf Acoust Speech Signal Proc 87-90, 1978.

12. Kates $\mathbb{M}$ : A computer simulation of hearing aid response and the effects of ear canal size. $J$ Acoust Soc Am 
Journal of Rehabilitation Research and Development Vol. 27 No. 3 Summer 1990

83(5):1952-1963, 1988.

13. Kates JM: A time-domain digital simulation of hearing aid response. J Rehabil Res Dev 27(3):279-294, 1990.

14. Kates JM: Signal processing for hearing aids. Hear Instrum 37(2):19-22, 1986.

15. Kryter KD: Methods for the calculation and use of the articulation index. J Acoust Soc Am 34:1689-1697, 1962.

16. McClellan JH, Parks TW, Rabiner LR: A computer program for designing optimum FIR linear phase digital filters. IEEE Trans Audio Electroacoust 21(6):506-526, 1973.

17. Pavlovic $\mathrm{CV}$ : Derivation of primary parameters and procedures for use in speech intelligibility predictions. $J$ Acoust Soc Am 82(2):413-422, 1987.

18. Peters RW, Burkhard MD: On noise distortion and harmonic distortion measurements. Industrial Research Products Report No. 10350-1. Elk Grove Village, IL: 1968.
19. Preves DA, Beck LB, Burnett ED, Teder H: Input stimuli for obtaining frequency responses of automatic gain control hearing aids. J Speech Hear Res 32:189-194, 1989.

20. Stearns SD: Tests of coherence unbiasing methods. IEEE Trans Acoust Speech Signal Proc 29:321-323, 1981.

21. Welch PD: The use of fast Fourier transform for the estimation of power spectra: A method based on time averaging over short, modified periodigrams. IEEE Trans Audio Electroacoust 15(2):70-73, 1967.

22. Widrow B, Glover JR Jr, McCool JM, Kaunitz J, Williams CS, Hearn $\mathrm{RH}$, Zeidler $\mathfrak{J R}$, Dong $\mathrm{E} J \mathrm{~J}$, Goodlin RC: Adaptive noise cancelling: Principles and applications. Proc IEEE 63(12):1692-1716, 1975.

23. Widrow B, McCool JM, Larimore MG, Johnson CR Jr: Nonstationary learning characteristics of the LMS adaptive filter. Proc IEEE 64(8):1151-1162, 1976. 


\section{APPENDIX A: SHAPED NOISE SIGNAL}

The broadband noise stimulus consists of white Gaussian noise passed through a high-pass filter at $200 \mathrm{~Hz}$ and a lowpass filter at $5000 \mathrm{~Hz}$ to restrict the bandwidth, and a one-pole low-pass filter at $900 \mathrm{~Hz}$ to give a speech-shaped spectrum. The high-pass and low-pass bandlimiting digital filters are derived from 3-pole analog Butterworth filters, the high-pass via the bilinear transformation and the low-pass via impulse invariance, and the one-pole analog filter at $900 \mathrm{~Hz}$ is also mapped into a digital filter via impulse invariance.

Define the analog filter frequencies in $\mathrm{Hz}$ :

$$
\begin{aligned}
& \mathrm{f}_{\mathrm{s}}=\text { sampling rate }(20000 \mathrm{~Hz}) \\
& \mathrm{f}_{\mathrm{h}}=\text { high-pass limiting frequency }(200 \mathrm{~Hz}) \\
& \mathrm{f}_{\mathrm{l}}=\text { low-pass limiting frequency }(5000 \mathrm{~Hz}) \\
& \mathrm{f}_{\mathrm{p}}=\text { low-pass speech-shaping frequency }(900 \mathrm{~Hz}) .
\end{aligned}
$$

The prewarped digital cutoff frequency for the high-pass filter is then

$$
\alpha=\tan \left(\pi f_{h} / f_{\mathrm{s}}\right) .
$$

The digital high-pass limiting filter (at $200 \mathrm{~Hz}$ ) is then, from the bilinear transformation:

$$
H(z)=\frac{1-3 z^{-1}+3 z^{-2}-z^{-3}}{a_{0}+a_{1} z^{-1}+a_{2} z^{-2}+a_{3} z^{-3}}
$$

where

$$
\begin{aligned}
& \mathrm{a}_{0}=(1+\alpha)\left(1+\alpha+\alpha^{2}\right) \\
& \mathrm{a}_{1}=(1+\alpha)\left(2 \alpha^{2}-2\right)+(\alpha-1)\left(1+\alpha+\alpha^{2}\right) \\
& \mathrm{a}_{2}=(\alpha-1)\left(2 \alpha^{2}-2\right)+(\alpha+1)\left(1-\alpha+\alpha^{2}\right) \\
& \mathrm{a}_{3}=(\alpha-1)\left(1-\alpha+\alpha^{2}\right) .
\end{aligned}
$$

In the time-domain, the filter becomes

$$
\begin{aligned}
y(n)= & {[x(n)-3 x(n-1)+3 x(n-2)-x(n-3)} \\
& \left.-a_{1} y(n-1)-a_{2} y(n-2)-a_{3} y(n-3)\right] / a_{0}
\end{aligned}
$$

where $x(n)$ is the filter input and $y(n)$ is the filter output.

The digital low-pass limiting filter (at $5000 \mathrm{~Hz}$ ) is then, via impulse invariance

$$
L(z)=g\left[\frac{1}{1-b_{0} z^{-1}}+\frac{-1+b_{1} z^{-1}}{1-b_{2} z^{-1}+b_{0} z^{-2}}\right]
$$

where

$$
\begin{aligned}
& \mathrm{b}_{0}=\exp \left(-2 \pi \mathrm{f}_{\mathrm{l}} / \mathrm{f}_{\mathrm{s}}\right) \\
& \mathrm{b}_{1}=\exp \left(-\pi \mathrm{f}_{\mathrm{l}} / \mathrm{f}_{\mathrm{s}}\right) \times \\
& \quad\left[\cos \left(\sqrt{3} \pi \mathrm{f}_{\mathrm{l}} / \mathrm{f}_{\mathrm{s}}\right)+\sin \left(\sqrt{3} \pi \mathrm{f}_{\mathrm{l}} / \mathrm{f}_{\mathrm{s}}\right) / \sqrt{3}\right] \\
& \mathrm{b}_{2}=2 \exp \left(-\pi \mathrm{f}_{\mathrm{l}} / \mathrm{f}_{\mathrm{s}}\right) \cos \left(\sqrt{3} \pi \mathrm{f}_{\mathrm{l}} / \mathrm{f}_{\mathrm{s}}\right) .
\end{aligned}
$$

The transfer function is normalized by $\mathrm{g}$ to get unity gain at low frequencies. In the time-domain, this filter is represented by

$$
\begin{aligned}
& y_{1}(n)=g x(n)+b_{0} y_{1}(n-1) \\
& y_{2}(n)=-g x(n)+g b_{1} x(n-1)+b_{2} y_{2}(n-1)-b_{0} y_{2}(n-2) \\
& y(n)=y_{1}(n)+y_{2}(n)
\end{aligned}
$$

where $x(n)$ is the filter input and $y(n)$ is the filter output.

The low-pass filter for shaping the speech spectrum is given by

$$
G(z)=\frac{\gamma_{0}}{1-\gamma_{1} z^{-1}}
$$

where

$$
\begin{aligned}
& \gamma_{0}=1-\exp \left(-2 \pi \mathrm{f}_{\mathrm{p}} / \mathrm{f}_{\mathrm{s}}\right) \\
& \gamma_{1}=1-\gamma_{0}
\end{aligned}
$$

In the time-domain, this filter becomes

$$
y(n)=\gamma_{0} x(n)+\gamma_{1} y(n-1)
$$

where $x(n)$ is the filter input and $y(n)$ is the filter output.

\section{APPENDIX B: SWEPT SINUSOID}

An assumption in testing a multiband hearing aid is that the frequency bands will tend to be uniformly spaced on a logarithmic frequency scale (typical of analog filter designs or approximations to auditory critical bands) as opposed to a linear frequency scale (typical of digital filter designs). The swept sinusoid should therefore spend equal amounts of time within equal logarithmic frequency bands.

Let

$$
\begin{aligned}
& \mathrm{N}=\text { duration of the sweep }(53248 \text { samples }) \\
& \mathrm{f}_{0}=\text { starting frequency for the sweep }(222 \mathrm{~Hz}) \\
& \mathrm{f}_{1}=\text { stopping frequency for the sweep }(4470 \mathrm{~Hz}) \\
& \mathrm{f}_{\mathrm{S}}=\text { sampling frequency }(20000 \mathrm{~Hz}) .
\end{aligned}
$$

The desired instantaneous frequency is given by

$$
\omega(n)=2 \pi\left(f_{0} / f_{s}\right) \exp (\xi n)
$$

where

$$
\zeta=\frac{1}{N-1} \ln \left(\frac{f_{1}}{f_{0}}\right) .
$$

The digital chirp signal for the frequency sweep is then

$$
\mathrm{s}(\mathrm{n})=\sin [\omega(\mathrm{n}) / \xi], 0 \leq \mathrm{n} \leq \mathrm{N}-1 .
$$

\section{APPENDIX C: ADAPTIVE CANCELLATION}

The adaptive cancellation system uses a pair of filters, each having $\mathrm{N}$ taps, as shown in Figure 2 . Let $\mathrm{s}(\mathrm{n})$ be the hearing aid response to the sweep alone, which is the reference input to adaptive filter $w(n)$. Let $r(n)$ be the hearing aid response to the shaped noise alone, which is the reference signal input to 
adaptive filter $v(n)$. The hearing aid response to the sweep plus noise is denoted by $x(n)$, and the response delayed by $N / 2$ samples is $\hat{x}(n)$. The error signal is given by $e(n)$, and the system adapts to drive $e(n)$ as close to zero as possible.

The power of the sweep-plus-noise signal changes with time because of the frequency dependent gain of the hearing aid. In order to maintain stability in the adaptive system, the adaptation time constant has been made dependent on the estimated signal power (11). The estimated signal power is given by

$$
\sigma^{2}(n)=\beta \sigma^{2}(n-1)+(1-\beta) \hat{x}^{2}(n)
$$

where

$$
\beta=\exp (-1 / 200)
$$

for the sampling rate of $20 \mathrm{kHz}$. The adaptation time constant is then given by

$$
\mu(\mathrm{n})=\frac{1}{4 N \sigma^{2}(\mathrm{n})}
$$

where $\mathrm{N}$ is the adaptive filter length in samples. A value of $\mathrm{N}$ $=15$ was used for the processing examples presented in this paper.

The error signal is given by

$$
e(n)=\hat{x}(n)-\sum_{k=0}^{N-1} w_{k}(n) s(n-k)-\sum_{k=0}^{N-1} v_{k}(n) r(n-k)
$$

and the updating of the adaptive filter coefficients (9) is then given by

$$
w_{k}(n)=w_{k}(n-1)+2 \mu(n) e(n) s(n-k)
$$

and

$$
v_{k}(n)=v_{k}(n-1)+2 \mu(n) e(n) r(n-k) .
$$

\section{APPENDIX D: COMB FILTER}

The shaped noise is convolved with a comb filter to produce the notched distortion test signal. Let $M$ be the number of valleys (or peaks) in the comb filter between $0 \mathrm{~Hz}$ and $\mathrm{f}_{\mathrm{s}} / 2$, the highest frequency that can be represented in the digital system. The filter was designed with $\mathrm{M}=16$. The comb is uniformly spaced on a linear frequency scale, and has unity gain for the peaks of the comb and $-62 \mathrm{~dB}$ gain for the valleys. The comb filter, designed using the Parks-McClellan algorithm (16), is given by

$$
H(z)=\sum_{k=0}^{30} h_{k} z^{-2 M k}
$$

where

$$
\begin{aligned}
& h_{0}=0.1476714 \times 10^{-2}=h_{30} \\
& \mathrm{~h}_{1}=0 \quad=\mathrm{h}_{29} \\
& h_{2}=-0.3777827 \times 10^{-2}=h_{28} \\
& \mathrm{~h}_{3}=0 \quad=\mathrm{h}_{27} \\
& \mathrm{~h}_{4}=0.8223298 \times 10^{-2}=\mathrm{h}_{26} \\
& \mathrm{~h}_{5}=0 \quad=\mathrm{h}_{25} \\
& \mathrm{~h}_{6}=-0.1578323 \times 10^{-1}=\mathrm{h}_{24} \\
& \mathrm{~h}_{7}=0 \quad=\mathrm{h}_{23} \\
& \mathrm{~h}_{8}=0.2832202 \times 10^{-1}=\mathrm{h}_{22} \\
& \mathrm{~h}_{9}=0 \quad=\mathrm{h}_{21} \\
& h_{10}=-0.5025136 \times 10^{-1}=h_{20} \\
& \mathrm{~h}_{11}=0 \quad=\mathrm{h}_{19} \\
& \mathrm{~h}_{12}=0.9755109 \times 10^{-1}=\mathrm{h}_{18} \\
& \mathrm{~h}_{13}=0 \quad=\mathrm{h}_{17} \\
& \mathrm{~h}_{14}=-0.3153704=\mathrm{h}_{16} \\
& \mathrm{~h}_{15}=0.5000000 \text {. }
\end{aligned}
$$

The comb filter in the time-domain is given by

$$
y(n)=\sum_{k=0}^{30} h_{k} x(n-2 M k)
$$

where $y(n)$ is the output of the filter and $x(n)$ the input. For $M=16$, the total filter length is 961 samples, of which only 17 are non-zero. 